\title{
Exact controllability of a piezoelectric body. Theory and numerical simulation
}

\author{
Bernadette Miara* and Arnaud Münch ${ }^{\dagger}$
}

August 28, 2008

\begin{abstract}
We study the exact controllability of a three-dimensional body made of a material whose constitutive law introduces an elasticity-electricity coupling. We show that a coupled elastic-electric control acting on the whole boundary of the body drives the system to rest after time large enough. Two-dimensional numerical experiments suggest that controllability can still be achieved by relaxing this restrictive condition using either both controls on a reduced support or elastic control alone.
\end{abstract}

Key words: Piezo-electricity, Exact boundary controllability, Finite element approximation.

Mathematics Subject Classification (2000): 35K05, 49Q20, 65M60.

\section{Introduction}

Piezoelectric materials are part of the family of "smart materials" (such as magnetostrictive materials, electro-rheological fluids, shape memory alloys). The electromechanical coupling they present (mechanical deformations in response to applied electric field and conversely dielectric polarization in response to strain) is used in the design of adaptive structures that can be monitored with large accuracy. For example their use as control elements is widespread: piezoelectric sensors or actuators patches are bonded to a surface, fibers are embedded within the structure to be controlled or stabilized (we refer to [22] for some applications of piezoelectric devices used in control). The problem under study here is the control of a body made of such a material, let us state it now.

Let $\Omega$ be a domain (connected, bounded subset) of $\mathbb{R}^{3}$ with regular boundary $\Gamma$ (this assumption will be made more precise later). Let $T>0$, we denote by $Q$ the domain $\Omega \times(0, T)$ and by $\Sigma$ its boundary, $\Sigma=\Gamma \times(0, T)$. The evolution equations satisfied by the elastic displacement field ${ }^{1} \boldsymbol{y}=\left(y_{i}\right): \bar{Q} \rightarrow \mathbb{R}^{3}$, and the scalar electric potential $\theta: \bar{Q} \rightarrow \mathbb{R}$

\footnotetext{
${ }^{*}$ Laboratoire de modélisation et simulation numérique, École Supérieure d’Ingénieurs en Électronique et Électrotechnique, 2 boulevard Blaise Pascal, 93160 Noisy-Le-Grand, France; e-mail: miarab@esiee.fr, Phone: +33-145926568, Fax: +33-145926699. Supported by the European project "INTAS, Research Project for South Caucasian Republics 06-1000017-8886".

${ }^{\dagger}$ Laboratoire de Mathématiques de Besançon, Université de Franche-Comté, UMR CNRS 6623, 16, route de Gray 25030 Besançon, France; e-mail : arnaud.munch@univ-fcomte.fr, Phone: +33-381 666 489, Fax: +33-381 666 623. Partially supported by grants ANR-05-JC-0182-01 and ANR-07-JC-1832-84.

${ }^{1}$ Latin exponents and indices take their values in the set $\{1,2,3\}$, Einstein convention for repeated exponents and indices is used and bold face letters represent vectors or vector spaces.
} 
of a piezoelectric body (without any volume electric charges or mechanical forces) under Cauchy initial conditions $\left(\boldsymbol{y}^{0}, \boldsymbol{y}^{1}\right)$ and Dirichlet boundary conditions $(\overline{\boldsymbol{y}}, \bar{\theta})$ reads

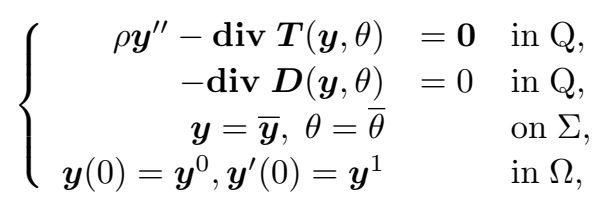

where $\rho$ is the mass density and where the stress tensor $\boldsymbol{T}=\left(T^{i j}\right)$ and the electric displacement $\boldsymbol{D}=\left(D^{i}\right)$ are related to the linearized deformation tensor and to the gradient of the electric potential through the constitutive law given in the next section, the symbol prime ' denotes the derivative with respect to time and div the divergence with respect to the space variables. In this work we address the question of the exact controllability, by a Dirichlet boundary action, of the solution of the piezoelectric problem introduced before. This controllability problem may be stated as follows: does it exists a minimal control time $T^{0}$, some initial conditions $\left(\boldsymbol{y}^{0}, \boldsymbol{y}^{1}\right)$ to be found in appropriate functional spaces and a boundary control $(\overline{\boldsymbol{y}}, \bar{\theta})$ such that the solution $\boldsymbol{y}=\boldsymbol{y}(t ; \overline{\boldsymbol{y}}, \bar{\theta})$ of problem (1) can be driven to equilibrium at time $T^{0}$, i.e.,

$$
\boldsymbol{y}(T ; \overline{\boldsymbol{y}}, \bar{\theta})=\boldsymbol{y}^{\prime}(T ; \overline{\boldsymbol{y}}, \bar{\theta})=\mathbf{0}, \quad \theta(T ; \overline{\boldsymbol{y}}, \bar{\theta})=0 \quad \text { in } \quad \Omega \text { for all } T \geq T^{0} .
$$

The method we use to investigate this question relies on the Hilbert Uniqueness Method [16] whose principle we recall now. First we consider the homogeneous evolution problem in $(\boldsymbol{u}, \varphi)$ with $\boldsymbol{u}^{0} \in \mathcal{D}(\Omega), \boldsymbol{u}^{1} \in \mathcal{D}(\Omega)$

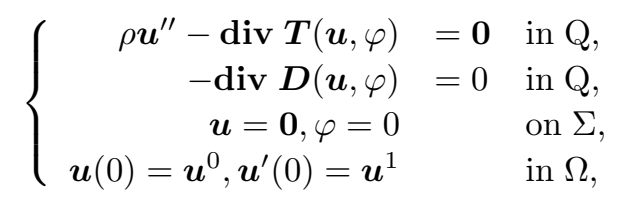

and the adjoint (backward, nonhomogeneous) problem in $(\boldsymbol{v}, \psi)$

$$
\left\{\begin{array}{rll}
\rho \boldsymbol{v}^{\prime \prime}-\operatorname{div} \boldsymbol{T}(\boldsymbol{v}, \psi) & =\mathbf{0} & \text { in } \mathrm{Q} \\
-\operatorname{div} \boldsymbol{D}(\boldsymbol{v}, \psi) & =0 & \text { in } \mathrm{Q} \\
\boldsymbol{v}=\overline{\boldsymbol{v}}(\boldsymbol{u}, \varphi), \psi=\bar{\psi}(\boldsymbol{u}, \varphi) & \text { on } \Sigma \\
\boldsymbol{v}(T)=\boldsymbol{v}^{\prime}(T)=\mathbf{0} & & \text { in } \Omega
\end{array}\right.
$$

Next we prove that, for an appropriate choice of the boundary conditions $(\overline{\boldsymbol{v}}(\boldsymbol{u}, \varphi), \bar{\psi}(\boldsymbol{u}, \varphi))$, there exists a functional space $\boldsymbol{F}$ and its dual space $\boldsymbol{F}^{\prime}$ such that for $T$ large enough, the linear operator

$$
\Lambda:\left(\boldsymbol{u}^{0}, \boldsymbol{u}^{1}\right) \in \mathcal{D}(\Omega) \times \mathcal{D}(\Omega) \rightarrow\left(\boldsymbol{v}^{\prime}(0),-\boldsymbol{v}(0)\right)
$$

can be extended to an operator (still denoted $\Lambda) \Lambda: \boldsymbol{F} \rightarrow \boldsymbol{F}^{\prime}$ continuous and coercive. Therefore by solving the equation $\Lambda\left(\boldsymbol{u}^{0}, \boldsymbol{u}^{1}\right)=\left(\boldsymbol{y}^{1},-\boldsymbol{y}^{0}\right),\left(\boldsymbol{y}^{1},-\boldsymbol{y}^{0}\right) \in \boldsymbol{F}^{\prime}$ we get first the unique initial conditions $\left(\boldsymbol{u}^{0}, \boldsymbol{u}^{1}\right) \in \boldsymbol{F}$ and next the boundary control $(\overline{\boldsymbol{y}}(\boldsymbol{u}, \varphi), \bar{\theta}(\boldsymbol{u}, \varphi))$ that drives the system to rest.

The plan of this paper is as follows: in Section 2 we recall the model of static piezoelectric bodies, in Section 3 we give the model of the evolution problem, in Section 4 we introduce the multipliers technique in order to get the so called "direct" and "strong " or "indirect" regularities of the solution of the homogeneous evolution problem, in Section 5 the conditions for exact controllability are obtained. Finally these theoretical results are illustrated, in a two 
dimensional situation, in Section 6, by numerical experiments applied to PZT piezoelectric material. We conclude this work with some remarks in Section 7.

The mathematical approach presented in that paper has first been exposed without details in [17]; extensions have also been obtained for layered materials [11, 12] as well as exact controllability for shells [18], stabilization by semi-group theory is under study [19]. We also refer the reader to $[6,7,23]$ for the controllability of so-called piezo-electric beams and to [3] for the optimal control of a piezo-electric shells model.

\section{The static problem}

We consider a piezoelectric body whose reference configuration $\bar{\Omega}$ is assumed to be stress-free and we denote by $\boldsymbol{x}=\left(x_{i}\right)$ a point in $\mathbb{R}^{3}$.

\subsection{The equilibrium equations}

Under the action of applied volume force $\boldsymbol{f}=\left(f_{i}\right): \bar{\Omega} \rightarrow \mathbb{R}^{3}$ and charges $\gamma: \bar{\Omega} \rightarrow \mathbb{R}$ the body undergoes an elastic displacement field $\boldsymbol{u}=\left(u_{i}\right): \bar{\Omega} \rightarrow \mathbb{R}^{3}$ and an electric potential $\varphi: \bar{\Omega} \rightarrow \mathbb{R}$. The couple $(\boldsymbol{u}, \varphi)$ solves the equilibrium equations

$$
\begin{cases}-\operatorname{div} \boldsymbol{T}(\boldsymbol{u}, \varphi)=\boldsymbol{f} & \text { in } \Omega \\ -\operatorname{div} \boldsymbol{D}(\boldsymbol{u}, \varphi)=\gamma & \text { in } \Omega\end{cases}
$$

with $(\operatorname{div} \boldsymbol{T})^{i}=\partial_{k} T^{k i}, \operatorname{div} \boldsymbol{D}=\partial_{k} D^{k}$. To avoid difficulties which are not relevant for this study we only consider homogeneous Dirichlet boundary conditions

$$
\boldsymbol{u}=\mathbf{0}, \varphi=0 \quad \text { on } \Gamma \text {. }
$$

\subsection{The constitutive law}

The properties of the material are described through three tensors. The fourth order tensor of elasticity $\left(c^{i j k l}\right)$ is symmetric and positive definite,

$$
c^{i j k l}=c^{j i k l}=c^{k l i j}, \quad \exists \alpha_{c}>0: c^{i j k l} X_{i j} X_{k l} \geq \alpha_{c} X_{i j} X_{i j}, \quad \forall X_{i j}=X_{j i} \in \mathbb{R} .
$$

The third order coupling tensor $\left(e^{i j k}\right)$ is symmetric, $e^{i j k}=e^{i k j}$. Finally, the second order dielectric tensor $\left(d^{i j}\right)$ is symmetric and positive definite,

$$
d^{i j}=d^{j i}, \quad \exists \alpha_{d}>0: d^{i j} X_{i} X_{j} \geq \alpha_{d} X_{i} X_{i}, \quad \forall X_{i} \in \mathbb{R} .
$$

In this paper the coefficients of these tensors are assumed to be independent of $\boldsymbol{x}$. Let $s_{k l}(\boldsymbol{u})=\left(\partial_{k} u_{l}+\partial_{l} u_{k}\right) / 2$ be the components of the linearized deformation tensor with $\partial_{k} u_{l}=\partial u_{l} / \partial x_{k}$. Therefore the constitutive law that relates the stress tensor $\boldsymbol{T}=\left(T^{i j}\right)$ and the electric displacement $\boldsymbol{D}=\left(D^{i}\right)$ to the linearized deformation tensor and to the gradient of the electric potential reads

$$
\left\{\begin{array}{l}
T^{i j}(\boldsymbol{u}, \varphi)=c^{i j k l} s_{k l}(\boldsymbol{u})+e^{k i j} \partial_{k} \varphi \text { in } \Omega \\
D^{i}(\boldsymbol{u}, \varphi)=-e^{i k l} s_{k l}(\boldsymbol{u})+d^{i j} \partial_{j} \varphi \text { in } \Omega
\end{array}\right.
$$




\subsection{Properties of the static solution}

We introduce the three bilinear functionals $c, e, d$ given by

$$
\left\{\begin{aligned}
c(\boldsymbol{u}, \boldsymbol{v}) & =c^{i j k l} s_{i j}(\boldsymbol{u}) s_{k l}(\boldsymbol{v}) \\
e(\boldsymbol{v}, \psi) & =e^{i j k} s_{j k}(\boldsymbol{v}) \partial_{i} \psi \\
d(\varphi, \psi) & =d^{i j} \partial_{i} \varphi \partial_{j} \psi
\end{aligned}\right.
$$

and the notation $\boldsymbol{u} \cdot \boldsymbol{v}=u_{i} v_{i},|\boldsymbol{v}|^{2}=\boldsymbol{v} \cdot \boldsymbol{v}$. We are now in a position to give the main properties of the solution to problem (2-3).

LEMmA 2.1 Let the boundary $\Gamma$ be Lipschitz-continuous and let $\boldsymbol{u} \in \boldsymbol{H}^{2}(\Omega), \varphi \in H^{2}(\Omega)$.

(i) For all test functions $\boldsymbol{v} \in \boldsymbol{H}^{1}(\Omega), \psi \in H^{1}(\Omega)$ we have ${ }^{2}$

$$
\begin{cases}-\int_{\Omega} \operatorname{div} \boldsymbol{T}(\boldsymbol{u}, \varphi) \cdot \boldsymbol{v} & =\int_{\Omega}(c(\boldsymbol{u}, \boldsymbol{v})+e(\boldsymbol{v}, \varphi))-\int_{\Gamma} T^{i j}(\boldsymbol{u}, \varphi) v_{j} n_{i} \\ -\int_{\Omega} \operatorname{div} \boldsymbol{D}(\boldsymbol{u}, \varphi) \psi & =\int_{\Omega}(-e(\boldsymbol{u}, \varphi)+d(\varphi, \psi))-\int_{\Gamma} D^{i}(\boldsymbol{u}, \varphi) \psi n_{i}\end{cases}
$$

(ii) For all test functions $\boldsymbol{v} \in \boldsymbol{H}_{0}^{1}(\Omega), \psi \in H_{0}^{1}(\Omega)$ we have

$$
\begin{cases}-\int_{\Omega} \operatorname{div} \boldsymbol{T}(\boldsymbol{u}, \varphi) \cdot \boldsymbol{v} & =\int_{\Omega}(c(\boldsymbol{u}, \boldsymbol{v})+e(\boldsymbol{v}, \varphi)) \\ -\int_{\Omega} \operatorname{div} \boldsymbol{D}(\boldsymbol{u}, \varphi) \psi & =\int_{\Omega}(-e(\boldsymbol{u}, \varphi)+d(\varphi, \psi)) .\end{cases}
$$

Proof of Lemma 2.1 - For part (i) we use Green formula and the definition of the bilinear functionals $c, e, d$ leading to

$$
\begin{aligned}
-\int_{\Omega} \operatorname{div} \boldsymbol{T}(\boldsymbol{u}, \varphi) \cdot \boldsymbol{v} & =\int_{\Omega} T^{i j}(\boldsymbol{u}, \varphi) \partial_{i} v_{j}-\int_{\Gamma} T^{i j}(\boldsymbol{u}, \varphi) v_{j} n_{i} \\
& =\int_{\Omega} T^{i j}(\boldsymbol{u}, \varphi) s_{i j}(\boldsymbol{v})-\int_{\Gamma} T^{i j}(\boldsymbol{u}, \varphi) v_{j} n_{i} \\
& =\int_{\Omega}(c(\boldsymbol{u}, \boldsymbol{v})+e(\boldsymbol{v}, \varphi))-\int_{\Gamma} T^{i j}(\boldsymbol{u}, \varphi) v_{j} n_{i}
\end{aligned}
$$

Similarly we get

$$
\begin{aligned}
-\int_{\Omega} \operatorname{div} \boldsymbol{D}(\boldsymbol{u}, \varphi) \psi & =\int_{\Omega} D^{i}(\boldsymbol{u}, \varphi) \partial_{i} \psi-\int_{\Gamma} D^{i}(\boldsymbol{u}, \varphi) \psi n_{i} \\
& =\int_{\Omega}(-e(\boldsymbol{u}, \varphi)+d(\varphi, \psi))-\int_{\Gamma} D^{i}(\boldsymbol{u}, \varphi) \psi n_{i} .
\end{aligned}
$$

The computation of part (ii) is obtained by taking into account the homogeneous Dirichlet boundary conditions.

The next Theorem states the conditions for a couple $(\boldsymbol{u}, \varphi)$ to be a strong or a weak solution to problem (2-3).

TheOREm 2.1 (i) Let the boundary $\Gamma$ be Lipschitz-continuous. For smooth data $\boldsymbol{f} \in \boldsymbol{L}^{2}(\Omega), \gamma \in$ $L^{2}(\Omega)$ there exists a unique weak solution $(\boldsymbol{u}, \varphi) \in \boldsymbol{H}_{0}^{1}(\Omega) \times H_{0}^{1}(\Omega)$ to the variational problem

$$
\left\{\begin{array}{lll}
\int_{\Omega}(c(\boldsymbol{u}, \boldsymbol{v})+e(\boldsymbol{v}, \varphi)) & =\int_{\Omega} \boldsymbol{f} \cdot \boldsymbol{v} & \forall \boldsymbol{v} \in \boldsymbol{H}_{0}^{1}(\Omega), \\
\int_{\Omega}(-e(\boldsymbol{u}, \psi)+d(\varphi, \psi)) & =\int_{\Omega}^{\gamma \psi} & \forall \psi \in H_{0}^{1}(\Omega) .
\end{array}\right.
$$

\footnotetext{
${ }^{2}$ For the sake of simplicity, measure terms $d x, d t, d \Gamma, \partial \Sigma$ are removed from the integrals expression.
} 
In addition this solution is the saddle-point of the static energy

$$
(\boldsymbol{v}, \psi) \in \boldsymbol{H}_{0}^{1}(\Omega) \times H_{0}^{1}(\Omega) \rightarrow \frac{1}{2} \int_{\Omega}(c(\boldsymbol{v}, \boldsymbol{v})+2 e(\boldsymbol{v}, \psi)-d(\psi, \psi))-\int_{\Omega} \boldsymbol{f} \cdot \boldsymbol{v}-\int_{\Omega} \gamma \psi
$$

(ii) Let $\Gamma$ be of class $C^{2}$. For regular data $\boldsymbol{f} \in \boldsymbol{H}^{1}(\Omega)$ and $\gamma \in H^{1}(\Omega)$, there exists a unique strong solution $\left(\boldsymbol{u} \in \boldsymbol{H}^{2}(\Omega) \cap \boldsymbol{H}_{0}^{1}(\Omega), \varphi \in H^{2}(\Omega) \cap H_{0}^{1}(\Omega)\right)$ to the variational problem

$$
\left\{\begin{array}{lll}
\int_{\Omega}(c(\boldsymbol{u}, \boldsymbol{v})+e(\boldsymbol{v}, \varphi)) & =\int_{\Omega} \boldsymbol{f} \cdot \boldsymbol{v} & \forall \boldsymbol{v} \in \boldsymbol{H}_{0}^{1}(\Omega), \\
\int_{\Omega}(-e(\boldsymbol{u}, \psi)+d(\varphi, \psi)) & =\int_{\Omega}^{\gamma \psi} \quad \forall \psi \in H_{0}^{1}(\Omega) .
\end{array}\right.
$$

Proof of Theorem 2.1- Due to the positivity of tensor $c^{i j k l}$ and Korn's inequality for elastic material the bilinear form $\int_{\Omega} c(\boldsymbol{u}, \boldsymbol{v}) d x$ is coercive on $\boldsymbol{H}_{0}^{1}(\Omega)$ and similarly by positivity of tensors $d^{i j}$ and Poincaré-Wirtinger's inequality, the bilinar form $\int_{\Omega} d(\varphi, \psi) d x$ is coercive on $H_{0}^{1}(\Omega)$. Hence the proof is a consequence of Lax-Milgram Theorem.

\section{The evolution problem}

The evolution problem of a three dimensional body made with a piezoelectric material with mass density $\rho>0$ reads

$$
\left\{\begin{array}{lll}
\rho \boldsymbol{u}^{\prime \prime} & -\operatorname{div} \boldsymbol{T}(\boldsymbol{u}, \varphi)=\mathbf{0} & \text { in } \mathrm{Q} \\
-\operatorname{div} \boldsymbol{D}(\boldsymbol{u}, \varphi)=0 & \text { in } \mathrm{Q},
\end{array}\right.
$$

with Dirichlet boundary conditions and Cauchy initial conditions

$$
\left\{\begin{array}{l}
\boldsymbol{u}=\overline{\boldsymbol{u}} \text { on } \Sigma^{0}, \varphi=\bar{\varphi} \text { on } \Sigma^{1} \\
\boldsymbol{u}(0)=\boldsymbol{u}^{0}, \boldsymbol{u}^{\prime}(0)=\boldsymbol{u}^{1} \text { in } \Omega
\end{array}\right.
$$

where $\boldsymbol{u}^{\prime \prime}=\partial^{2} \boldsymbol{u} / \partial t^{2}$. We assume in the sequel that the mass density is constant and without loss of generality $\rho=1$, thus the associated homogeneous evolution problem reads: Find $\boldsymbol{u}:(\boldsymbol{x}, t) \in \bar{Q} \longrightarrow \mathbb{R}^{3}, \varphi:(\boldsymbol{x}, t) \in \bar{Q} \longrightarrow \mathbb{R}$ such that

$$
\left\{\begin{array}{rll}
\boldsymbol{u}^{\prime \prime}-\operatorname{div} \boldsymbol{T}(\boldsymbol{u}, \varphi) & =\mathbf{0} & \text { in } \mathrm{Q} \\
-\operatorname{div} \boldsymbol{D}(\boldsymbol{u}, \varphi) & =0 & \text { in } \mathrm{Q} \\
\boldsymbol{u}=\mathbf{0}, \varphi=0 & \text { on } \Sigma \\
\boldsymbol{u}(0)=\boldsymbol{u}^{0}, \boldsymbol{u}^{\prime}(0)=\boldsymbol{u}^{1} & \text { in } \Omega
\end{array}\right.
$$

As mentioned in the Introduction we have to consider the evolution problem in both cases with homogeneous and with nonhomogeneous boundary conditions; their properties are given in the next section.

\subsection{The homogeneous problem}

LEMMA 3.1 (i) For $\Gamma$ of class $C^{2}$ and $\boldsymbol{u}^{0} \in \boldsymbol{H}^{2}(\Omega) \cap \boldsymbol{H}_{0}^{1}(\Omega), \boldsymbol{u}^{1} \in \boldsymbol{H}_{0}^{1}(\Omega)$, the system (4) has a unique strong solution

$$
\boldsymbol{u} \in \boldsymbol{C}\left(0, T ; \boldsymbol{H}^{2}(\Omega) \cap \boldsymbol{H}_{0}^{1}(\Omega)\right), \quad \boldsymbol{u}^{\prime} \in \boldsymbol{C}\left(0, T ; \boldsymbol{H}_{0}^{1}(\Omega)\right), \quad \varphi \in C\left(0, T ; H^{2}(\Omega) \cap H_{0}^{1}(\Omega)\right) .
$$


(ii) For $\Gamma$ Lipschitz-continuous and $\left(\boldsymbol{u}^{0} \in \boldsymbol{H}_{0}^{1}(\Omega), \boldsymbol{u}^{1} \in \boldsymbol{L}^{2}(\Omega)\right)$, system (4) has a unique weak solution

$$
\boldsymbol{u} \in \boldsymbol{C}\left(0, T ; \boldsymbol{H}_{0}^{1}(\Omega)\right), \quad \boldsymbol{u}^{\prime} \in \boldsymbol{C}\left(0, T ; \boldsymbol{L}^{2}(\Omega)\right), \quad \varphi \in C\left(0, T ; H_{0}^{1}(\Omega)\right) .
$$

Proof of Lemma 3.1 - This is a direct consequence of Lions "abstract" theorem [15].

\subsection{The nonhomogeneous problem}

Let us consider the nonhomogeneous problem

$$
\left\{\begin{array}{rll}
\boldsymbol{v}^{\prime \prime}-\operatorname{div} \boldsymbol{T}(\boldsymbol{v}, \psi) & =\mathbf{0} & \text { in } \mathrm{Q}, \\
-\operatorname{div} \boldsymbol{D}(\boldsymbol{v}, \psi) & =0 & \text { in } \mathrm{Q} \\
\boldsymbol{v}=\overline{\boldsymbol{v}}, \psi=\bar{\psi} & & \text { on } \Sigma, \\
\boldsymbol{v}(0)=\boldsymbol{v}^{0}, \boldsymbol{v}^{\prime}(0)=\boldsymbol{v}^{1} & & \text { in } \Omega
\end{array}\right.
$$

and the associate reverse homogeneous problem

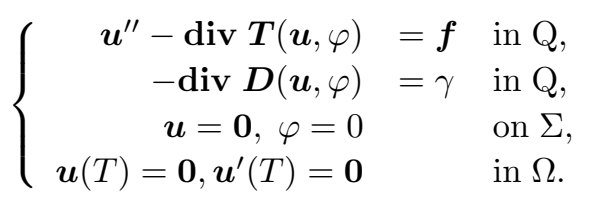

The weak solution to this problem is given by the transposition method [16] as follows: Formally, we multiply the first equation of (6) by $\boldsymbol{v}$ and the second one by $\psi$ and similarly we multiply the first equation of (5) by $\boldsymbol{u}$ and the second one by $\varphi$. Integration over $Q=\Omega \times(0, T)$ then leads to

$$
\begin{aligned}
& \int_{Q} \boldsymbol{f}(t) \cdot \boldsymbol{v}(t)+\gamma(t) \psi(t)=\int_{\Omega} \boldsymbol{u}^{0} \cdot \boldsymbol{v}^{1}-\boldsymbol{u}^{1} \cdot \boldsymbol{v}^{0}-\int_{\Sigma}\left(c^{i j k l} s_{k l}(\boldsymbol{u})+e^{k i j} \partial_{k} \varphi\right) \bar{v}_{j} n_{i} \\
&+\left(e^{i k l} s_{k l}(\boldsymbol{u})-d^{i j} \partial_{j} \varphi\right) \bar{\psi} n_{i}
\end{aligned}
$$

Let $\boldsymbol{F}$ be a functional space and $\boldsymbol{F}^{\prime}$ its dual space, for $\boldsymbol{U}(0)=\left(\boldsymbol{u}^{0}, \boldsymbol{u}^{1}\right) \in \boldsymbol{F}, \boldsymbol{V}(0)=$ $\left(\boldsymbol{v}^{1},-\boldsymbol{v}^{0}\right) \in \boldsymbol{F}^{\prime}$ we note $<\boldsymbol{U}(0), \boldsymbol{V}(0)>=\int_{\Omega} \boldsymbol{u}^{0} \cdot \boldsymbol{v}^{1}-\boldsymbol{u}^{1} \cdot \boldsymbol{v}^{0}$ where $<., .>$ is the duality between $\boldsymbol{F}$ and $\boldsymbol{F}^{\prime}$. The couple $(\boldsymbol{v}, \psi)$ is called a weak solution to (5) if, for initial condition $\boldsymbol{V}(0) \in \boldsymbol{F}^{\prime}$ and boundary condition $\left.\left(\overline{\boldsymbol{v}} \in \boldsymbol{L}^{2}(\Sigma)\right), \bar{\psi} \in L^{2}(\Sigma)\right)$, it satisfies (7) for all $\boldsymbol{f} \in \mathcal{D}(\Omega)$ and $\gamma \in \mathcal{D}(\Omega)$. Thus we can state for example the following result for the nonhomogeneous problem (5) associated to the strong solution to (6) with $\boldsymbol{u}^{0} \in \boldsymbol{H}^{2}(\Omega) \cap \boldsymbol{H}_{0}^{1}(\Omega), \boldsymbol{u}^{1} \in \boldsymbol{H}_{0}^{1}(\Omega)$.

Lemma 3.2 Let $\Gamma$ be of class $C^{2}$. For all $\boldsymbol{v}^{0} \in \boldsymbol{H}^{-1}(\Omega), \boldsymbol{v}^{1} \in\left(\boldsymbol{H}^{2}(\Omega) \cap \boldsymbol{H}_{0}^{1}(\Omega)\right)^{\prime}, \overline{\boldsymbol{v}} \in \boldsymbol{L}^{2}(\Sigma)$ and $\bar{\psi} \in E^{2}(\Sigma)$, there exists a unique solution

$$
\boldsymbol{v} \in \boldsymbol{L}^{\infty}\left(0, T ; \boldsymbol{H}^{-1}(\Omega)\right), \quad \boldsymbol{v}^{\prime} \in \boldsymbol{L}^{\infty}\left(0, T ;\left(\boldsymbol{H}^{2}(\Omega) \cap \boldsymbol{H}_{0}^{1}(\Omega)\right)^{\prime}\right), \quad \psi \in L^{\infty}\left(0, T ; H^{-1}(\Omega)\right) .
$$




\section{Properties of the homogeneous evolution problem}

We continue the investigation of the properties of the solution of the homogeneous problem, namely that the energy of the weak solution is constant over its trajectory.

\subsection{Energy and Maupertuis' principle}

LEMMA 4.1 Let $\boldsymbol{u}$ be the weak solution of problem (4).

(i) When no mechanical forces or electric charges are applied, the energy

$$
E(t)=\frac{1}{2} \int_{\Omega}\left|\boldsymbol{u}^{\prime}(t)\right|^{2}+c(\boldsymbol{u}(t), \boldsymbol{u}(t))+d(\varphi(t), \varphi(t)) \quad t \geq 0
$$

is constant along each trajectory, i.e.,

$$
E(t)=E^{0}=\frac{1}{2} \int_{\Omega}\left|\boldsymbol{u}^{1}\right|^{2}+c\left(\boldsymbol{u}^{0}, \boldsymbol{u}^{0}\right)+d\left(\varphi^{0}, \varphi^{0}\right)
$$

where $\varphi^{0} \in H_{0}^{1}(\Omega)$ is the unique weak solution to $\int_{\Omega} d\left(\varphi^{0}, \psi\right)=\int_{\Omega} e\left(\boldsymbol{u}^{0}, \psi\right) \quad \forall \psi \in H_{0}^{1}(\Omega)$.

(ii) The weak solution satisfies Maupertuis' principle:

$$
\int_{Q}\left|\boldsymbol{u}^{\prime}(t)\right|^{2}-c(\boldsymbol{u}(t), \boldsymbol{u}(t))-d(\varphi(t), \varphi(t))=\left.\int_{\Omega}\left(\boldsymbol{u}^{\prime}(t) \cdot \boldsymbol{u}(t)\right)\right|_{0} ^{T}
$$

with the notation $\left.v\right|_{0} ^{T}=v(T)-v(0)$.

Proof of Lemma 4.1 - We first prove step $(i)$ for strong solution. We use Lemma 2.1 (ii) with test-functions $\boldsymbol{v}=\boldsymbol{u}^{\prime}$ to get

$$
-\int_{\Omega} \operatorname{div} \boldsymbol{T}(\boldsymbol{u}, \varphi) \cdot \boldsymbol{u}^{\prime}=\int_{\Omega} c\left(\boldsymbol{u}, \boldsymbol{u}^{\prime}\right)+e\left(\boldsymbol{u}^{\prime}, \varphi\right) .
$$

Then we differentiate with respect to time the second equation of problem (4) and use similarly Lemma 2.1 (ii) with test-function $\psi=\varphi$

$$
0=-\int_{\Omega} \operatorname{div} \boldsymbol{D}\left(\boldsymbol{u}^{\prime}, \varphi^{\prime}\right) \varphi=\int_{\Omega}-e\left(\boldsymbol{u}^{\prime}, \varphi\right)+d\left(\varphi, \varphi^{\prime}\right) .
$$

Hence

$$
-\int_{\Omega} \operatorname{div} \boldsymbol{T}(\boldsymbol{u}, \varphi) \cdot \boldsymbol{u}^{\prime}=\int_{\Omega} c\left(\boldsymbol{u}, \boldsymbol{u}^{\prime}\right)+d\left(\varphi, \varphi^{\prime}\right)=\frac{1}{2} \frac{d}{d t} \int_{\Omega} c(\boldsymbol{u}, \boldsymbol{u})+d(\varphi, \varphi) .
$$

Finally an integration by parts yields

$$
0=\int_{\Omega}\left(\boldsymbol{u}^{\prime \prime}-\operatorname{div} \boldsymbol{T}(\boldsymbol{u}, \varphi)\right) \cdot \boldsymbol{u}^{\prime}=\frac{1}{2} \frac{d}{d t} \int_{\Omega}\left|\boldsymbol{u}^{\prime}\right|^{2}+c(\boldsymbol{u}, \boldsymbol{u})+d(\varphi, \varphi)=\frac{d}{d t} E(t) .
$$

Next when $\boldsymbol{u}$ is a weak solution we get the result by a density argument: let $\left(\boldsymbol{u}_{k}^{0}, \boldsymbol{u}_{k}^{1}\right) \in$ $\boldsymbol{H}^{2}(\Omega) \cap \boldsymbol{H}_{0}^{1}(\Omega) \times \boldsymbol{H}_{0}^{1}(\Omega)$ be a sequence of Cauchy conditions such that $\left(\boldsymbol{u}_{k}^{0}, \boldsymbol{u}_{k}^{1}\right) \longrightarrow$ $\left(\boldsymbol{u}^{0}, \boldsymbol{u}^{1}\right)$ in $\boldsymbol{H}_{0}^{1}(\Omega) \times \boldsymbol{L}^{2}(\Omega)$ and let $\left(\boldsymbol{u}_{k}, \varphi_{k}\right)$ be the associated strong solution. From the continuous dependence of the solution with respect to the initial data, we have $\boldsymbol{u}_{k} \longrightarrow$ 
$\boldsymbol{u}$ in $\boldsymbol{C}\left(0, \mathrm{~T} ; \boldsymbol{H}_{0}^{1}(\Omega)\right) \cap \boldsymbol{C}^{1}\left(0, \mathrm{~T} ; \boldsymbol{L}^{2}(\Omega)\right)$ and $\varphi_{k} \longrightarrow \varphi$ in $C\left(0, T ; H_{0}^{1}(\Omega)\right) \cap C^{1}\left(0, T ; L^{2}(\Omega)\right)$. Hence

$E_{k}(t)=\frac{1}{2} \int_{\Omega}\left|\boldsymbol{u}_{k}^{\prime}\right|^{2}+c\left(\boldsymbol{u}_{k}, \boldsymbol{u}_{k}\right)+d\left(\varphi_{k}, \varphi_{k}\right) \longrightarrow E(t)=\frac{1}{2} \int_{\Omega}\left|\boldsymbol{u}^{\prime}\right|^{2}+c(\boldsymbol{u}, \boldsymbol{u})+d(\varphi, \varphi)$ in $C(0, T)$.

The step (ii) or Maupertuis principle is obtained by multiplying the first equation of (4) by $\boldsymbol{u}$, the second one by $\varphi$, by integrating over $Q$ and taking into account the homogeneous Dirichlet boundary conditions

$$
\begin{aligned}
0=\int_{Q}\left(\boldsymbol{u}^{\prime \prime}-\operatorname{div} \boldsymbol{T}(\boldsymbol{u}, \varphi)\right) \cdot \boldsymbol{u} & =\int_{Q}-\left|\boldsymbol{u}^{\prime}\right|^{2}+c(\boldsymbol{u}, \boldsymbol{u})+e(\boldsymbol{u}, \varphi)-\int_{\Gamma} T^{i j}(\boldsymbol{u}, \varphi) u_{j} n_{i}+\left.\int_{\Omega}\left(\boldsymbol{u}^{\prime} \cdot \boldsymbol{u}\right)\right|_{0} ^{T}, \\
& =\int_{Q}-\left|\boldsymbol{u}^{\prime}\right|^{2}+c(\boldsymbol{u}, \boldsymbol{u})+e(\boldsymbol{u}, \varphi)+\left.\int_{\Omega}\left(\boldsymbol{u}^{\prime} \cdot \boldsymbol{u}\right)\right|_{0} ^{T}, \\
0=-\int_{\Omega} \operatorname{div} \boldsymbol{D}(\boldsymbol{u}, \varphi) \varphi & =\int_{\Omega}-e(\boldsymbol{u}, \varphi)+d(\varphi, \varphi)-\int_{\Gamma} D^{i}(\boldsymbol{u}, \varphi) \varphi n_{i}=\int_{\Omega}-e(\boldsymbol{u}, \varphi)+d(\varphi, \varphi),
\end{aligned}
$$

and therefore

$0=\int_{Q}\left(\boldsymbol{u}^{\prime \prime}-\operatorname{div} \boldsymbol{T}(\boldsymbol{u}, \varphi)\right) \cdot \boldsymbol{u}-\int_{\Omega} \operatorname{div} \boldsymbol{D}(\boldsymbol{u}, \varphi) \varphi=\int_{Q}-\left|\boldsymbol{u}^{\prime}\right|^{2}+c(\boldsymbol{u}, \boldsymbol{u})+d(\varphi, \varphi)+\left.\int_{\Omega}\left(\boldsymbol{u}^{\prime} \cdot \boldsymbol{u}\right)\right|_{0} ^{T} \cdot$

\subsection{Direct and reverse identities}

We introduce the classical multipliers technique to get the observability condition.

Lemma 4.2 (i) For any field $\boldsymbol{q}=\left(q_{m}\right) \in \boldsymbol{C}^{2}(\bar{\Omega})$ the weak solution satisfies the "direct" identity

$$
\begin{aligned}
\frac{1}{2} \int_{\Sigma}(\boldsymbol{q} \cdot \boldsymbol{n})(c(\boldsymbol{u}, \boldsymbol{u})+ & 2 e(\boldsymbol{u}, \varphi)-d(\varphi, \varphi))= \\
& \frac{1}{2} \int_{Q} \partial_{m} q_{m}\left(\left|\boldsymbol{u}^{\prime}\right|^{2}-c(\boldsymbol{u}, \boldsymbol{u})-2 e(\boldsymbol{u}, \varphi)+d(\varphi, \varphi)\right)+\left.\int_{\Omega}\left(\boldsymbol{u}^{\prime} \cdot q_{m} \partial_{m} \boldsymbol{u}\right)\right|_{0} ^{T} \\
& +\int_{Q}\left(c^{i j k l} s_{i j}(\boldsymbol{u})+e^{i k l} \partial_{i} \varphi\right) g_{k l}(\boldsymbol{q}, \boldsymbol{u})+\left(e^{k i j} s_{i j}(\boldsymbol{u})-d^{i k} \partial_{i} \varphi\right) \partial_{k} q_{m} \partial_{m} \varphi
\end{aligned}
$$

where $g_{k l}(\boldsymbol{q}, \boldsymbol{u})=\frac{1}{2}\left(\partial_{k} q_{m} \partial_{m} u_{l}+\partial_{l} q_{m} \partial_{m} u_{k}\right)$ and $\boldsymbol{n}$ is the unit normal vector.

(ii) For $\boldsymbol{x}^{\mathbf{0}} \in \mathbb{R}^{3}$ and $\boldsymbol{p}(\boldsymbol{x})=\boldsymbol{x}-\boldsymbol{x}^{\mathbf{0}}$ the weak solution satisfies the "reverse" identity

$$
\frac{1}{2} \int_{\Sigma}(\boldsymbol{p} \cdot \boldsymbol{n})(c(\boldsymbol{u}, \boldsymbol{u})+2 e(\boldsymbol{u}, \varphi)-d(\varphi, \varphi))=T E^{0}+\left.\int_{\Omega}\left(\boldsymbol{u}^{\prime} \cdot\left(p_{m} \partial_{m} \boldsymbol{u}+\boldsymbol{u}\right)\right)\right|_{0} ^{T} .
$$

Proof of Lemma 4.2- The direct identity is obtained first for strong solution and then for the weak solution with a density argument. Let us multiply the first equation (4) by the multiplier $q_{m} \partial_{m} \boldsymbol{u}$ and the second one by $q_{m} \partial_{m} \varphi$. We first prove $(i)$. The proof is broken into 5 steps.

- Step 1. . Let $\boldsymbol{u}$ be a strong solution to (4).We have

$$
\int_{Q} \boldsymbol{u}^{\prime \prime} \cdot q_{m} \partial_{m} \boldsymbol{u}=\frac{1}{2} \int_{Q} \partial_{m} q_{m}\left|\boldsymbol{u}^{\prime}\right|^{2}+\left.\int_{\Omega}\left(\boldsymbol{u}^{\prime} \cdot q_{m} \partial_{m} \boldsymbol{u}\right)\right|_{0} ^{T} .
$$


This result is obtained by an integration by parts and by using the boundary condition $\boldsymbol{u}^{\prime}(t)=\mathbf{0}$ on $\Gamma($ since $\boldsymbol{u}(t)=\mathbf{0}$ on $\Gamma$ for all $t \geq 0)$, therefore we get

$$
\begin{aligned}
\int_{Q} \boldsymbol{u}^{\prime \prime} \cdot q_{m} \partial_{m} \boldsymbol{u} & =\left.\int_{\Omega}\left(\boldsymbol{u}^{\prime} \cdot q_{m} \partial_{m} \boldsymbol{u}\right)\right|_{0} ^{T}-\int_{Q} \boldsymbol{u}^{\prime} \cdot q_{m} \partial_{m} \boldsymbol{u}^{\prime} \\
\int_{\Omega} \boldsymbol{u}^{\prime} \cdot q_{m} \partial_{m} \boldsymbol{u}^{\prime} & =\frac{1}{2} \int_{\Omega} q_{m} \partial_{m}\left|\boldsymbol{u}^{\prime}\right|^{2}=-\frac{1}{2} \int_{\Omega} \partial_{m} q_{m}\left|\boldsymbol{u}^{\prime}\right|^{2}+\frac{1}{2} \int_{\Gamma}(\boldsymbol{q} \cdot \boldsymbol{n})\left|\boldsymbol{u}^{\prime}\right|^{2}=-\frac{1}{2} \int_{\Omega} \partial_{m} q_{m}\left|\boldsymbol{u}^{\prime}\right|^{2}
\end{aligned}
$$

- Step 2. For all $\boldsymbol{q} \in \boldsymbol{C}^{2}(\bar{\Omega})$ and $\boldsymbol{v} \in \boldsymbol{H}^{2}(\Omega)$, we have

$$
s_{k l}\left(q_{m} \partial_{m} \boldsymbol{v}\right)=q_{m} \partial_{m} s_{k l}(\boldsymbol{v})+g_{k l}(\boldsymbol{q}, \boldsymbol{v}) .
$$

To show this result let us first consider a scalar multiplier $q \in C^{1}(\bar{\Omega})$ and $\boldsymbol{w}=\left(w_{l}\right) \in \boldsymbol{H}^{1}(\Omega)$ we get

$$
s_{k l}(q \boldsymbol{w})=\frac{1}{2}\left(\partial_{k}(q \boldsymbol{w})_{l}+\partial_{l}(q \boldsymbol{w})_{k}\right)=q s_{k l}(\boldsymbol{w})+\frac{1}{2}\left(w_{l} \partial_{k} q+w_{k} \partial_{l} q\right)
$$

A direct computation gives $s_{k l}\left(\partial_{m} \boldsymbol{v}\right)=\partial_{m} s_{k l}(\boldsymbol{v})$ for all $\boldsymbol{v} \in \boldsymbol{C}^{2}(\bar{\Omega})$, and Step 2 is obtained by letting $q=q_{m}, \boldsymbol{w}=\partial_{m} \boldsymbol{v}$.

- Step 3. For all $\boldsymbol{q} \in \boldsymbol{C}^{2}(\bar{\Omega})$ and $\boldsymbol{v} \in \boldsymbol{H}_{0}^{1}(\Omega), \psi \in H_{0}^{1}(\Omega)$, we have

$$
\left\{\begin{aligned}
\int_{\Omega} c\left(\boldsymbol{v}, q_{m} \partial_{m} \boldsymbol{v}\right) & =\int_{\Omega}\left(-\frac{1}{2} \partial_{m} q_{m} c(\boldsymbol{v}, \boldsymbol{v})+c^{i j k l} s_{i j}(\boldsymbol{v}) g_{k l}(\boldsymbol{q}, \boldsymbol{v})\right)+\frac{1}{2} \int_{\Gamma}(\boldsymbol{q} \cdot \boldsymbol{n}) c(\boldsymbol{v}, \boldsymbol{v}), \\
\int_{\Omega} e\left(\boldsymbol{v}, q_{m} \partial_{m} \psi\right) & =\int_{\Omega} e^{i j k} s_{j k}(\boldsymbol{v})\left(q_{m} \partial_{m i} \psi+\partial_{i} q_{m} \partial_{m} \psi\right), \\
\int_{\Omega} e\left(q_{m} \partial_{m} \boldsymbol{v}, \psi\right) & =\int_{\Omega}\left(-\partial_{m} q_{m} e(\boldsymbol{v}, \psi)-e^{i j k} q_{m} s_{k l}(\boldsymbol{v}) \partial_{i m} \psi+e^{i j k} g_{k l}(\boldsymbol{q}, \boldsymbol{v}) \partial_{i} \psi\right) \\
& +\int_{\Gamma}(\boldsymbol{q} \cdot \boldsymbol{n}) e(\boldsymbol{v}, \psi), \\
\int_{\Omega} d\left(\psi, q_{m} \partial_{m} \psi\right) & =-\frac{1}{2} \int_{\Omega} \partial_{m} q_{m} d(\psi, \psi)+\int_{\Omega} d^{i j} \partial_{i} \psi \partial_{j} q_{m} \partial_{m} \psi+\frac{1}{2} \int_{\Gamma}(\boldsymbol{q} \cdot \boldsymbol{n}) d(\psi, \psi) .
\end{aligned}\right.
$$


The result is obtained by easy computations:

$$
\begin{aligned}
\int_{\Omega} c\left(\boldsymbol{v}, q_{m} \partial_{m} \boldsymbol{v}\right) & =\int_{\Omega} c^{i j k l} s_{i j}(\boldsymbol{v}) s_{k l}\left(q_{m} \partial_{m} \boldsymbol{v}\right) \\
& =\int_{\Omega} q_{m} c^{i j k l} s_{i j}(\boldsymbol{v}) \partial_{m} s_{k l}(\boldsymbol{v})+c^{i j k l} s_{i j}(\boldsymbol{v}) g_{k l}(\boldsymbol{q}, \boldsymbol{v}) \\
& =\int_{\Omega} \frac{1}{2} q_{m} \partial_{m} c(\boldsymbol{v}, \boldsymbol{v})+c^{i j k l} s_{i j}(\boldsymbol{v}) g_{k l}(\boldsymbol{q}, \boldsymbol{v}), \\
& =\int_{\Omega}-\frac{1}{2} \partial_{m} q_{m} c(\boldsymbol{v}, \boldsymbol{v})+c^{i j k l} s_{i j}(\boldsymbol{v}) g_{k l}(\boldsymbol{q}, \boldsymbol{v})+\frac{1}{2} \int_{\Gamma}(\boldsymbol{q} \cdot \boldsymbol{n}) c(\boldsymbol{v}, \boldsymbol{v}), \\
\int_{\Omega} e\left(\boldsymbol{v}, q_{m} \partial_{m} \psi\right) & =\int_{\Omega} e^{i j k} s_{j k}(\boldsymbol{v}) \partial_{i}\left(q_{m} \partial_{m} \psi\right)=\int_{\Omega} e^{i j k} s_{j k}(\boldsymbol{v})\left(q_{m} \partial_{m i} \psi+\partial_{i} q_{m} \partial_{m} \psi\right), \\
\int_{\Omega} e\left(q_{m} \partial_{m} \boldsymbol{v}, \psi\right) & =\int_{\Omega} e^{i k l} s_{k l}\left(q_{m} \partial_{m} \boldsymbol{v}\right) \partial_{i} \psi=\int_{\Omega} e^{i k l}\left(q_{m} \partial_{m} s_{k l}(\boldsymbol{v})+g_{k l}(\boldsymbol{q}, \boldsymbol{v})\right) \partial_{i} \varphi \\
& =\partial_{m}(\boldsymbol{q} \cdot \boldsymbol{n}) e(\boldsymbol{v}, \psi), \\
\int_{\Omega} d\left(\psi, q_{m} \partial_{m} \psi\right) & =\int_{\Omega} d^{i j} \partial_{i} \psi \partial_{j}\left(q_{m} \partial_{m} \psi\right),=\int_{\Omega} d^{i j} \partial_{i} \psi\left(q_{m} \partial_{m j} \psi+\partial_{j} q_{m} \partial_{m} \psi\right), \\
& =\int_{\Omega} \frac{1}{2} q_{m} \partial_{m} d(\psi, \psi)+\int_{\Omega} d^{i j} \partial_{i} \psi \partial_{j} q_{m} \partial_{m} \psi \\
& =-\int_{\Omega} \frac{1}{2} \partial_{m} q_{m} d\left(\psi, \psi \partial_{i m} \psi+e^{i j k} q_{m} g_{k l}(\boldsymbol{q}, \boldsymbol{u}) \partial_{i} \psi\right)
\end{aligned}
$$

- Step 4. For all strong solution associated to initial conditions $\left(\boldsymbol{u}^{0}, \boldsymbol{u}^{1}\right) \in \boldsymbol{H}^{2}(\Omega) \cap \boldsymbol{H}_{0}^{1}(\Omega) \times$ $\boldsymbol{H}_{0}^{1}(\Omega)$ and for all $\boldsymbol{q} \in \boldsymbol{C}^{2}(\bar{\omega})$, we have

$$
\begin{aligned}
\left.\int_{\Omega}-\operatorname{div} \boldsymbol{T}(\boldsymbol{u}, \varphi)\right) \cdot q_{m} \partial_{m} \boldsymbol{u}= & -\frac{1}{2} \int_{\Gamma}(\boldsymbol{q} \cdot \boldsymbol{n})(c(\boldsymbol{u}, \boldsymbol{u})+2 e(\boldsymbol{u}, \varphi)-d(\varphi, \varphi)) \\
& +\frac{1}{2} \int_{\Omega}-\partial_{m} q_{m}(c(\boldsymbol{u}, \boldsymbol{u})+2 e(\boldsymbol{u}, \varphi)-d(\varphi, \varphi)) \\
& +\int_{\Omega}\left(c^{i j k l} s_{i j}(\boldsymbol{u}) g_{k l}(\boldsymbol{q}, \boldsymbol{u})+e^{i j k} s_{j k}(\boldsymbol{u}) \partial_{i} q_{m} \partial_{m} \varphi-d^{i j} \partial_{i} \varphi \partial_{j} q_{m} \partial_{m} \varphi\right) .
\end{aligned}
$$

Proof. Since $(\boldsymbol{u}, \varphi)$ is a strong solution of (4) we can use Lemma 2.1(i) with $\boldsymbol{v}=q_{m} \partial_{m} \boldsymbol{u}$ and $\psi=q_{m} \partial_{m} \varphi$. Therefore

$$
\begin{aligned}
\int_{\Omega}-\operatorname{div} \boldsymbol{T}(\boldsymbol{u}, \varphi) & \cdot q_{m} \partial_{m} \boldsymbol{u}=\int_{\Omega}\left(c\left(\boldsymbol{u}, q_{m} \partial_{m} \boldsymbol{u}\right)+e\left(q_{m} \partial_{m} \boldsymbol{u}, \varphi\right)\right)-\int_{\Gamma} T^{i j}(\boldsymbol{u}, \varphi) q_{m} \partial_{m} u_{j} n_{i} \\
= & \int_{\Omega}\left(-\frac{1}{2} \partial_{m} q_{m} c(\boldsymbol{u}, \boldsymbol{u})+c^{i j k l} s_{i j}(\boldsymbol{u}) g_{k l}(\boldsymbol{q}, \boldsymbol{u})-\partial_{m} q_{m} e(\boldsymbol{u}, \varphi)-e^{i j k} q_{m} s_{j k}(\boldsymbol{u}) \partial_{i m} \varphi\right) \\
& +\int_{\Gamma}(\boldsymbol{q} \cdot \boldsymbol{n})\left(\frac{1}{2} c(\boldsymbol{u}, \boldsymbol{u})+e(\boldsymbol{u}, \varphi)\right)-\int_{\Gamma} T^{i j}(\boldsymbol{u}, \varphi) q_{m} \partial_{m} u_{j} n_{i},
\end{aligned}
$$

and since

$$
\begin{aligned}
0=\int_{\Omega}-\operatorname{div} \boldsymbol{D}(\boldsymbol{u}, \varphi) q_{m} \partial_{m} \varphi= & \int_{\Omega}-e\left(\boldsymbol{u}, q_{m} \partial_{m} \varphi\right)+d\left(\varphi, q_{m} \partial_{m} \varphi\right)-\int_{\Gamma} D^{i}(\boldsymbol{u}, \varphi) q_{m} \partial_{m} \varphi n_{i}, \\
= & \int_{\Omega}-e^{i j k} s_{j k}(\boldsymbol{u})\left(q_{m} \partial_{m i} \varphi+\partial_{i} q_{m} \partial_{m} \varphi\right)-\int_{\Omega} \frac{1}{2} \partial_{m} q_{m} d(\varphi, \varphi) \\
& +\int_{\Omega} d^{i j} \partial_{i} \varphi \partial_{j} q_{m} \partial_{m} \varphi+\int_{\Gamma} \frac{1}{2}(\boldsymbol{q} \cdot \boldsymbol{n}) d(\varphi, \varphi)-\int_{\Gamma} D^{i}(\boldsymbol{u}, \varphi) q_{m} \partial_{m} \varphi n_{i},
\end{aligned}
$$


we finally obtain

$$
\begin{aligned}
\left.\int_{\Omega}-\operatorname{div} \boldsymbol{T}(\boldsymbol{u}, \varphi)\right) \cdot q_{m} \partial_{m} \boldsymbol{u}= & \frac{1}{2} \int_{\Omega}-\partial_{m} q_{m}(c(\boldsymbol{u}, \boldsymbol{u})+2 e(\boldsymbol{u}, \varphi)-d(\varphi, \varphi) \\
& +\int_{\Omega} c^{i j k l} s_{i j}(\boldsymbol{u}) g_{k l}(\boldsymbol{q}, \boldsymbol{u})+e^{i j k} s_{j k}(\boldsymbol{u}) \partial_{i} q_{m} \partial_{m} \varphi-d^{i j} \partial_{i} \varphi \partial_{j} q_{m} \partial_{m} \varphi \\
& +\frac{1}{2} \int_{\Gamma}(\boldsymbol{q} \cdot \boldsymbol{n})(c(\boldsymbol{u}, \boldsymbol{u})+2 e(\boldsymbol{u}, \varphi)-d(\varphi, \varphi)) \\
& -\int_{\Gamma} T^{i j}(\boldsymbol{u}, \varphi) q_{m} \partial_{m} u_{j} n_{i}+D^{i}(\boldsymbol{u}, \varphi) q_{m} \partial_{m} \varphi n_{i} .
\end{aligned}
$$

Since $\boldsymbol{u}=\mathbf{0}$ on $\Gamma$, then $\partial_{m} \boldsymbol{u}=n_{m} \partial_{\boldsymbol{n}} \boldsymbol{u}$ and $q_{m} \partial_{m} \boldsymbol{u}=(\boldsymbol{q} \cdot \boldsymbol{n}) \partial_{\boldsymbol{n}} \boldsymbol{u}$ or component-wise $q_{m} \partial_{m} u_{j} n_{i}=(\boldsymbol{q} \cdot \boldsymbol{n}) \partial_{i} u_{j}$ on $\Gamma$. This implies

$T^{i j}(\boldsymbol{u}, \varphi) q_{m} \partial_{m} u_{j} n_{i}=(\boldsymbol{q} \cdot \boldsymbol{n}) T^{i j}(\boldsymbol{u}, \varphi) \partial_{i} u_{j}=(\boldsymbol{q} \cdot \boldsymbol{n}) T^{i j}(\boldsymbol{u}, \varphi) s_{i j}(\boldsymbol{u})=(\boldsymbol{q} \cdot \boldsymbol{n})(c(\boldsymbol{u}, \boldsymbol{u})+e(\boldsymbol{u}, \varphi))$ on $\Gamma$.

Similarly $\varphi=0$ on $\Gamma$ implies $D^{i}(\boldsymbol{u}, \varphi) q_{m} \partial_{m} \varphi n_{i}=(\boldsymbol{q} \cdot \boldsymbol{n})(-e(\boldsymbol{u}, \varphi)+d(\varphi, \varphi))$ on $\Gamma$, thus the first equality is obtained for strong solution.

- Step 5 . The equality is then established by density for the weak solution.

Proof of (ii). By taking $\boldsymbol{q}=\boldsymbol{p}=\boldsymbol{x}-\boldsymbol{x}_{\mathbf{0}}$ we get $g_{k l}(\boldsymbol{p}, \boldsymbol{u})=s_{k l}(\boldsymbol{u}$ ) (let us note that $\partial_{m} p_{i}=\delta_{m}^{i}$ and $\partial_{m} p_{m}=3$ in dimension 3$)$. Therefore

$$
\begin{aligned}
\frac{1}{2} \int_{\Sigma}(\boldsymbol{p} \cdot \boldsymbol{n})(c(\boldsymbol{u}, \boldsymbol{u})+2 e(\boldsymbol{u}, \varphi)-d(\varphi, \varphi))= & \frac{3}{2} \int_{Q}\left(\left|\boldsymbol{u}^{\prime}\right|^{2}-c(\boldsymbol{u}, \boldsymbol{u})-2 e(\boldsymbol{u}, \varphi)+d(\varphi, \varphi)\right) \\
& +\left.\int_{\Omega}\left(\boldsymbol{u}^{\prime} \cdot \partial_{m} p_{m} \boldsymbol{u}\right)\right|_{0} ^{T}+\int_{Q}(c(\boldsymbol{u}, \boldsymbol{u})+2 e(\boldsymbol{u}, \varphi)-d(\varphi, \varphi)) .
\end{aligned}
$$

Since $0=\int_{Q} e(\boldsymbol{u}, \varphi)-d(\varphi, \varphi)$ for all weak solution to the homogeneous problem we obtain

$$
\begin{aligned}
\frac{1}{2} \int_{\Sigma}(\boldsymbol{p} \cdot \boldsymbol{n}) & (c(\boldsymbol{u}, \boldsymbol{u})+2 e(\boldsymbol{u}, \varphi)-d(\varphi, \varphi)) \\
& =\frac{1}{2} \int_{Q}\left(\left|\boldsymbol{u}^{\prime}\right|^{2}+c(\boldsymbol{u}, \boldsymbol{u})+d(\varphi, \varphi)\right)+\int_{Q}\left(\left|\boldsymbol{u}^{\prime}\right|^{2}-c(\boldsymbol{u}, \boldsymbol{u})-d(\varphi, \varphi)\right)+\left.\int_{\Omega}\left(\boldsymbol{u}^{\prime} \cdot \partial_{m} p_{m} \boldsymbol{u}\right)\right|_{0} ^{T} \\
& =T E^{0}+\int_{Q}\left(\left|\boldsymbol{u}^{\prime}\right|^{2}-c(\boldsymbol{u}, \boldsymbol{u})-d(\varphi, \varphi)\right)+\left.\int_{\Omega}\left(\boldsymbol{u}^{\prime} \cdot \partial_{m} p_{m} \boldsymbol{u}\right)\right|_{0} ^{T}
\end{aligned}
$$

and we get the result thanks to Maupertuis' principle.

\subsection{Uniqueness Theorem for the homogeneous evolution problem}

In this section we establish other regularity conditions (the strong direct and reverse inequalities and consequently the hidden regularity and observability condition) for the weak solution of the homogeneous piezoelectric problem.

THeOREM 4.1 Let the boundary $\Gamma$ be of class $C^{2}$.

(i) There exists a positive constant $K(\boldsymbol{q}, \bar{\Omega})$ such that weak solution satisfies the "direct inequality"

$$
\left|\int_{\Sigma}(\boldsymbol{q} \cdot \boldsymbol{n})(c(\boldsymbol{u}, \boldsymbol{u})+2 e(\boldsymbol{u}, \varphi)-d(\varphi, \varphi))\right| \leq K(\boldsymbol{q}, \bar{\Omega})(T+1) E^{0}
$$


(ii) This implies the "hidden regularity": The quantity $\left|\int_{\Sigma} c(\boldsymbol{u}, \boldsymbol{u})+2 e(\boldsymbol{u}, \varphi)-d(\varphi, \varphi)\right|$ is bounded.

Proof of Theorem 4.1.- Lemma $4.2(i)$ yields

$$
\begin{aligned}
\mid \int_{\Sigma}(\boldsymbol{q} \cdot \boldsymbol{n})(c(\boldsymbol{u}, \boldsymbol{u})+ & 2 e(\boldsymbol{u}, \varphi)-d(\varphi, \varphi))|\leq| \int_{Q} \partial_{m} q_{m}\left(\left|\boldsymbol{u}^{\prime}\right|^{2}-c(\boldsymbol{u}, \boldsymbol{u})-2 e(\boldsymbol{u}, \varphi)+d(\varphi, \varphi)\right) \mid \\
& +2\left|\int_{Q}\left(c^{i j k l} s_{i j}(\boldsymbol{u})+e^{i k l} \partial_{i} \varphi\right) g_{k l}(\boldsymbol{q}, \boldsymbol{u})+\left(e^{k i j} s_{i j}(\boldsymbol{u})-d^{i k} \partial_{i} \varphi\right) \partial_{k} q_{m} \partial_{m} \varphi\right| \\
& +2\left|\int_{\Omega}\left(\boldsymbol{u}^{\prime} \cdot q_{m} \partial_{m} \boldsymbol{u}\right)\right|_{0}^{T} \mid
\end{aligned}
$$

Since $\boldsymbol{q} \in C^{2}(\Omega)$ we note $K_{1}(\boldsymbol{q}, \bar{\Omega})$ a positive bound that depends only upon $\boldsymbol{q}$ and $\bar{\Omega}$ such that

$$
\sup _{\boldsymbol{x} \in \bar{\Omega} ;(i, m) \in\{1,3\}}\left\{\left|q_{i}(\boldsymbol{x})\right|,\left|\partial_{m} q_{i}(\boldsymbol{x})\right|\right\} \leq K_{1}(\boldsymbol{q}, \bar{\Omega}) .
$$

Let us introduce the notations

$$
|v|_{0}=\left(\int_{\Omega}|v|^{2}\right)^{\frac{1}{2}}, \quad|v|_{1}=\left(\sum_{m \in\{1,3\}} \int_{\Omega}\left|\partial_{m} v\right|^{2}\right)^{\frac{1}{2}} .
$$

Poincaré-Wirtinger inequality states that for all $v \in H^{1}(\Omega)$ there exists a constant $K_{P}(\Omega)$ such that

$$
|v|_{0} \leq K_{P}(\Omega)|v|_{1} \quad \forall v \in H^{1}(\Omega)
$$

Korn's inequality for elastic materials states that there exists a constant $K_{K}(\Omega)$ such that for all $\boldsymbol{v} \in \boldsymbol{H}_{0}^{1}(\Omega)$

$$
\|\boldsymbol{v}\|_{\boldsymbol{H}^{1}(\Omega)} \leq K_{K}(\Omega)\left(\sum_{k, l \in\{1,3\}} \int_{\Omega}\left|s_{k l}(\boldsymbol{v})\right|^{2}\right)^{\frac{1}{2}} .
$$

Therefore by using the coercivity property of both tensors $c^{i j k l}$ and $d^{i j}$ (introduced in Section 2.2 ) we can establish an upper bound for each term of the right-hand side of (8): The two first terms are bounded by $K_{2}(\Omega) T E^{0}$, the last one is bounded by $2 K_{3}(\Omega) E^{0}$ and hence the global bound follows. Next, according to Komornik ([13], p.18), there exists a vector field $\boldsymbol{q} \in \boldsymbol{C}^{2}(\bar{\Omega})$ such that $\boldsymbol{q}=\boldsymbol{n}$ on $\Gamma$. With this choice of multiplier the hidden regularity is obtained.

Remark 1 This is a weaker condition than the one obtained for pure elastic body which is $c(\boldsymbol{u}, \boldsymbol{u}) \in L^{1}(\Sigma)$ or, for the classical wave equation, $\partial_{\boldsymbol{n}} \boldsymbol{u} \in \boldsymbol{L}^{2}(\Sigma)$. It comes from the fact that in the elastic case the solution minimizes the energy, in the piezoelectric case the solution is a saddle point of the energy.

THEOREM 4.2 Let the boundary $\Gamma$ be of class $C^{2}$. There exists a minimum time $T^{0}=$ $T^{0}\left(\boldsymbol{x}^{\mathbf{0}}, \Omega\right)$ such that for all $T \geq T^{0}$ the weak solution satisfies the "inverse inequality"

$$
E^{0}\left(T-T^{0}\right) \leq \frac{1}{2} \int_{\Sigma}(\boldsymbol{p} \cdot \boldsymbol{n})(c(\boldsymbol{u}, \boldsymbol{u})+2 e(\boldsymbol{u}, \varphi)-d(\varphi, \varphi)) .
$$


Proof of Theorem 4.2- It is broken into 2 steps

- Step 1. We first show that there exists a constant $\alpha=\alpha(\Omega)$ (which depends only upon $\Omega$ ) such that

$$
\int_{\Omega} \boldsymbol{u}^{\prime} \cdot\left(p_{m} \partial_{m} \boldsymbol{u}+\boldsymbol{u}\right) \leq \alpha E^{0}
$$

We follow [16]: For any constant $\beta>0$, we have

$$
2\left|\int_{\Omega} \boldsymbol{u}^{\prime} \cdot\left(p_{m} \partial_{m} \boldsymbol{u}+\boldsymbol{u}\right)\right| \leq \int_{\Omega}\left(\beta\left|\boldsymbol{u}^{\prime}\right|^{2}+\frac{1}{\beta}\left|p_{m} \partial_{m} \boldsymbol{u}+\boldsymbol{u}\right|^{2}\right) .
$$

Recalling that $\boldsymbol{u}=\mathbf{0}$ on the boundary we have

$$
\int_{\Omega} p_{m} \partial_{m} \boldsymbol{u} \cdot \boldsymbol{u}=\frac{1}{2} \int_{\Omega} p_{m} \partial_{m}|\boldsymbol{u}|^{2}=-\frac{1}{2} \int_{\Omega} \partial_{m} p_{m}|\boldsymbol{u}|^{2}+\frac{1}{2} \int_{\Gamma}(\boldsymbol{p} \cdot \boldsymbol{n})|\boldsymbol{u}|^{2}=-\frac{3}{2} \int_{\Omega}|\boldsymbol{u}|^{2}
$$

and we obtain

$$
\begin{aligned}
\int_{\Omega}\left|p_{m} \partial_{m} \boldsymbol{u}+\boldsymbol{u}\right|^{2} & =\int_{\Omega}\left(\left|p_{m} \partial_{m} \boldsymbol{u}\right|^{2}+|\boldsymbol{u}|^{2}+2 p_{m} \partial_{m} \boldsymbol{u} \cdot \boldsymbol{u}\right)=\int_{\Omega}\left(\left|p_{m} \partial_{m} \boldsymbol{u}\right|^{2}-2|\boldsymbol{u}|^{2}\right) \\
& \leq \int_{\Omega}\left|p_{m} \partial_{m} \boldsymbol{u}\right|^{2} \leq 3 R^{2} \int_{\Omega}|\nabla \boldsymbol{u}|^{2}
\end{aligned}
$$

where $R=\max _{\boldsymbol{x} \in \bar{\Omega}}\left|\boldsymbol{x}-\boldsymbol{x}^{\mathbf{0}}\right|$. We use the same argument (Poincaré-Wirtinger and Korn's inequalities) as in the proof of the previous Theorem to get

$$
\int_{\Omega}\left|p_{m} \partial_{m} \boldsymbol{u}+\boldsymbol{u}\right|^{2} \leq 3 R^{2} K_{K}(\Omega) \int_{\Omega} c(\boldsymbol{u}, \boldsymbol{u}) \leq 3 R^{2} K_{K}(\Omega) E^{0}
$$

Therefore

$$
2\left|\int_{\Omega} \boldsymbol{u}^{\prime} \cdot\left(p_{m} \partial_{m} \boldsymbol{u}+\boldsymbol{u}\right)\right| \leq \int_{\Omega}\left(\beta\left|\boldsymbol{u}^{\prime}\right|^{2}+\frac{3 R^{2} K_{K}(\Omega)}{\beta} c(\boldsymbol{u}, \boldsymbol{u})\right) .
$$

With the choice $\beta=\sqrt{3 K_{K}(\Omega)} R$ we get

$$
2\left|\int_{\Omega} \boldsymbol{u}^{\prime} \cdot\left(p_{m} \partial_{m} \boldsymbol{u}+\boldsymbol{u}\right)\right| \leq \beta \int_{\Omega}\left(\left|\boldsymbol{u}^{\prime}\right|^{2}+c(\boldsymbol{u}, \boldsymbol{u})\right) \leq 2 \beta E^{0}
$$

and thereby the result with $\alpha=\beta$.

- Step 2. We rewrite the identity of Lemma 4.2 (ii)

$$
\frac{1}{2} \int_{\Sigma}(\boldsymbol{p} \cdot \boldsymbol{n})(c(\boldsymbol{u}, \boldsymbol{u})+2 e(\boldsymbol{u}, \varphi)-d(\varphi, \varphi))=T E^{0}+\left.\int_{\Omega}\left(\boldsymbol{u}^{\prime} \cdot\left(p_{m} \partial_{m} \boldsymbol{u}+\boldsymbol{u}\right)\right)\right|_{0} ^{T}
$$

and use the bound obtained in Step 1, $\left|\int_{\Omega}\left(\boldsymbol{u}^{\prime} \cdot\left(p_{m} \partial_{m} \boldsymbol{u}+\boldsymbol{u}\right)\right)\right|_{0}^{T}|\leq 2| \int_{\Omega}\left(\boldsymbol{u}^{\prime} \cdot\left(p_{m} \partial_{m} \boldsymbol{u}+\right.\right.$ $\boldsymbol{u})) \mid \leq 2 \alpha E^{0}$. Hence by letting $T^{0}=2 \alpha$ we obtain

$$
\frac{1}{2} \int_{\Sigma}(\boldsymbol{p} \cdot \boldsymbol{n})(c(\boldsymbol{u}, \boldsymbol{u})+2 e(\boldsymbol{u}, \varphi)-d(\varphi, \varphi)) \geq\left(T-T^{0}\right) E^{0}
$$


Remark 2 The sign of the quantity $c(\boldsymbol{v}, \boldsymbol{v})+2 e(\boldsymbol{v}, \psi)-d(\psi, \psi)$ is not known for any $\boldsymbol{v}$ and $\psi$, hence the uniqueness result is of this special form: If, for all $T \geq T^{0}$, the weak solution satisfies the following property on $\Sigma$

$$
\left\{\begin{array}{c}
c(\boldsymbol{u}, \boldsymbol{u})+2 e(\boldsymbol{u}, \varphi)-d(\varphi, \varphi) \leq 0 \text { when } \boldsymbol{p} \cdot \boldsymbol{n} \geq 0 \\
c(\boldsymbol{u}, \boldsymbol{u})+2 e(\boldsymbol{u}, \varphi)-d(\varphi, \varphi) \geq 0 \text { when } \boldsymbol{p} \cdot \boldsymbol{n} \leq 0
\end{array}\right.
$$

then $\boldsymbol{u}=\mathbf{0}$ and $\varphi=0$ in $\Omega$.

The inverse inequality is now used to derive other uniqueness properties of the solution to the homogeneous evolution problem.

Corollary 1 ( $i)$ Let $\Omega$ be a sphere with center $\boldsymbol{x}^{\mathbf{0}}$ and radius $R$. Then there exists a minimum time $T^{0}$ such that for all $T \geq T^{0}$ the weak solution satisfies the "inverse inequality"

$$
E^{0}\left(T-T^{0}\right)=\frac{R}{2} \int_{\Sigma} c(\boldsymbol{u}, \boldsymbol{u})+2 e(\boldsymbol{u}, \varphi)-d(\varphi, \varphi) .
$$

This implies that if, for all $T \geq T^{0}, c(\boldsymbol{u}, \boldsymbol{u})+2 e(\boldsymbol{u}, \varphi)-d(\varphi, \varphi) \leq 0$ on $\Sigma$ then $\boldsymbol{u}=0$ and $\varphi=0$ in $\Omega$.

(ii) Let $\Omega$ be $x^{0}$-star shaped, i.e., $\boldsymbol{p}(\boldsymbol{x}) \cdot \boldsymbol{n}(\boldsymbol{x})>0$ for all points $\boldsymbol{x} \in \Gamma$. Then there exists a minimum time $T^{0}$ and a constant $K$ (which depends upon the data $\left(c^{i j k l}, e^{i j k}, d^{i j}\right)$ ) such that for all $T \geq T^{0}$ the weak solution satisfies the "inverse inequality"

$$
E^{0}\left(T-T^{0}\right) \leq K R \int_{\Sigma} c(\boldsymbol{u}, \boldsymbol{u})
$$

with $R=\max _{\boldsymbol{x} \in \Omega}\left|\boldsymbol{x}-\boldsymbol{x}^{\mathbf{0}}\right|$.

For regular solution having a trace $c(\boldsymbol{u}, \boldsymbol{u}) \in L^{1}(\Sigma)$ this implies that if, for all $T \geq T^{0}$, $c(\boldsymbol{u}, \boldsymbol{u})=0$ on $\Sigma$ then $\boldsymbol{u}=0$ and $\varphi=0$ in $\Omega$.

(iii) Let us consider the partition $\Gamma=\Gamma^{+} \cup \Gamma^{-}, \Gamma^{+} \cap \Gamma^{-}=\emptyset$ with $\boldsymbol{p}(\boldsymbol{x}) \cdot \boldsymbol{n}(\boldsymbol{x})>0$ for all points $\boldsymbol{x} \in \Gamma^{+}$and $\boldsymbol{p}(\boldsymbol{x}) \cdot \boldsymbol{n}(\boldsymbol{x}) \leq 0$ for all points $\boldsymbol{x} \in \Gamma^{-}$, we note $\Sigma^{+}=\Gamma^{+} \times(0, T)$, $\Sigma^{+}=\Gamma^{+} \times(0, T)$.

There exists a minimum time $T^{0}$ such that for all $T \geq T^{0}$ the weak solution satisfies another "inverse inequality"

$$
\left(T-T^{0}\right) E^{0} \leq K R\left(\int_{\Sigma^{+}} c(\boldsymbol{u}, \boldsymbol{u})+\int_{\Sigma^{-}} d(\varphi, \varphi)\right),
$$

where the constant $K$ depends upon the data $\left(c^{i j k l}, e^{i j k}, d^{i j}\right)$.

For regular solution having traces $c(\boldsymbol{u}, \boldsymbol{u}) \in L^{1}\left(\Sigma^{+}\right), d(\varphi, \varphi) \in L^{1}\left(\Sigma^{-}\right)$this implies that if, for all $T \geq T^{0}, c(\boldsymbol{u}, \boldsymbol{u})=0$ on $\Sigma^{+}$and $d(\varphi, \varphi)=0$ on $\Sigma^{-}$then $\boldsymbol{u}=\mathbf{0}$ and $\varphi=0$ in $\Omega$.

- Proof of $(i)$. For a sphere $R=\left|\boldsymbol{x}-\boldsymbol{x}^{\mathbf{0}}\right|, \boldsymbol{x} \in \Gamma$, we have $\boldsymbol{p} \cdot \boldsymbol{n}=R$, hence the result. The uniqueness property is of the same kind as the one of Remark 2.

- Proof of $($ ii $)$. Let us adapt the algebraic inequality

$$
\forall \alpha>0, \quad 2|\boldsymbol{v} \cdot \boldsymbol{w}| \leq \alpha|\boldsymbol{v}|^{2}+\frac{1}{\alpha}|\boldsymbol{w}|^{2} \quad \forall \boldsymbol{v}, \boldsymbol{w} \in \mathbb{R}^{3}
$$

in the following way: There exists two positive constants $\alpha>0, \beta>0$ so that

$$
2|e(\boldsymbol{v}, \psi)| \leq \alpha c(\boldsymbol{v}, \boldsymbol{v})+\beta d(\psi, \psi) \quad \forall \boldsymbol{v} \in \boldsymbol{H}^{1}(\Omega), \psi \in H^{1}(\Omega)
$$


and we adjust these constants $\alpha, \beta$ to show that there exist two positive constants $K_{1}, K_{2}$ (that depend upon the data $\left.\left(c^{i j k l}, e^{i j k}, d^{i j}\right)\right)$ such that

$$
-K_{1} d(\varphi, \varphi) \leq c(\boldsymbol{u}, \boldsymbol{u})+2 e(\boldsymbol{u}, \varphi)-d(\varphi, \varphi) \leq K_{2} c(\boldsymbol{u}, \boldsymbol{u}) .
$$

Hence

$$
\int_{\Sigma}(\boldsymbol{p} \cdot \boldsymbol{n})(c(\boldsymbol{u}, \boldsymbol{u})+2 e(\boldsymbol{u}, \varphi)-d(\varphi, \varphi)) \leq K_{2} \int_{\Sigma}(\boldsymbol{p} \cdot \boldsymbol{n}) c(\boldsymbol{u}, \boldsymbol{u}) \leq K_{2} R \int_{\Sigma} c(\boldsymbol{u}, \boldsymbol{u}) .
$$

- Proof of (iii). We make use of the partition $\Sigma=\Sigma^{+} \cup \Sigma^{-}$to obtain

$$
\begin{aligned}
\int_{\Sigma}(\boldsymbol{p} \cdot \boldsymbol{n})(c(\boldsymbol{u}, \boldsymbol{u})+2 e(\boldsymbol{u}, \varphi)-d(\varphi, \varphi))= & \int_{\Sigma^{+}}(\boldsymbol{p} \cdot \boldsymbol{n})(c(\boldsymbol{u}, \boldsymbol{u})+2 e(\boldsymbol{u}, \varphi)-d(\varphi, \varphi)) \\
& +\int_{\Sigma^{-}}(-\boldsymbol{p} \cdot \boldsymbol{n})(-c(\boldsymbol{u}, \boldsymbol{u})-2 e(\boldsymbol{u}, \varphi)+d(\varphi, \varphi)) .
\end{aligned}
$$

As in the previous step,

$$
\int_{\Sigma^{+}}(\boldsymbol{p} \cdot \boldsymbol{n})(c(\boldsymbol{u}, \boldsymbol{u})+2 e(\boldsymbol{u}, \varphi)-d(\varphi, \varphi)) \leq K_{2} \int_{\Sigma^{+}}(\boldsymbol{p} \cdot \boldsymbol{n}) c(\boldsymbol{u}, \boldsymbol{u}) \leq K_{2} R \int_{\Sigma^{+}} c(\boldsymbol{u}, \boldsymbol{u})
$$

and

$\int_{\Sigma^{-}}(-\boldsymbol{p} \cdot \boldsymbol{n})(-c(\boldsymbol{u}, \boldsymbol{u})-2 e(\boldsymbol{u}, \varphi)+d(\varphi, \varphi)) \leq K_{1} \int_{\Sigma^{-}}(-\boldsymbol{p} \cdot \boldsymbol{n}) d(\varphi, \varphi) \leq K_{1} R \int_{\Sigma^{-}} d(\varphi, \varphi)$.

Remark 3 The restriction to regular solutions in steps (ii) and (iii) is due to the fact that there is no traces $c(\boldsymbol{u}, \boldsymbol{u}), d(\varphi, \varphi)$ in $L^{1}(\Sigma)$ for weak solutions.

\section{$5 \quad$ Exact controllability}

We are now in a position to state our controllability result. We follow the scheme detailed in the Introduction. The evolution problem reads: Find the elastic displacement field $\boldsymbol{y}$ and the electric potential $\theta$ governed by the hyperbolic equations

$$
\left\{\begin{array}{rll}
\boldsymbol{y}^{\prime \prime}-\operatorname{div} \boldsymbol{T}(\boldsymbol{y}, \theta) & =\mathbf{0} & \text { in } \mathrm{Q}, \\
-\operatorname{div} \boldsymbol{D}(\boldsymbol{y}, \theta) & =0 & \text { in } \mathrm{Q} \\
\boldsymbol{y}=\overline{\boldsymbol{y}}, \theta=\bar{\theta} & & \text { on } \Sigma, \\
\boldsymbol{y}(0)=\boldsymbol{y}^{0}, \boldsymbol{y}^{\prime}(0)=\boldsymbol{y}^{1} & & \text { in } \Omega .
\end{array}\right.
$$

In the next two sections we consider the adjoint reverse problem in $(\boldsymbol{v}, \psi)$ and show how to compute the boundary conditions $(\overline{\boldsymbol{y}}, \bar{\theta})$ to drive the system to rest.

\subsection{The adjoint backward problem}

Associated to the solution $(\boldsymbol{u}, \varphi)$ of problem (4) with smooth initial conditions $\left(\boldsymbol{u}^{0}, \boldsymbol{u}^{1}\right) \in$ $\mathcal{D}(\Omega) \times \mathcal{D}(\Omega)$ we consider the adjoint backward and nonhomogeneous problem in $(\boldsymbol{v}, \psi)$ (with nonhomogeneous boundary Dirichlet conditions depending on $(\boldsymbol{u}, \varphi$ ) and final Cauchy conditions) 


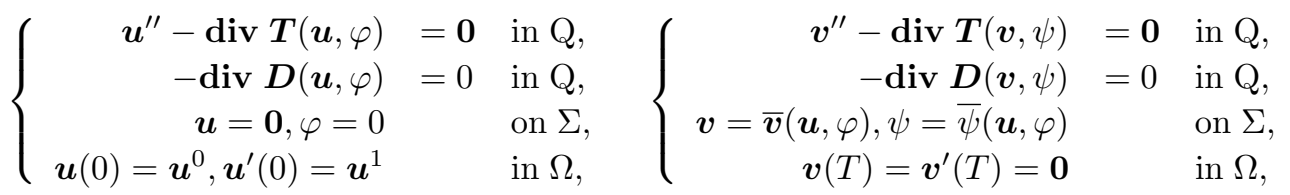

the dependence $\overline{\boldsymbol{v}}(\boldsymbol{u}, \varphi)$ and $\bar{\psi}(\boldsymbol{u}, \varphi)$ will be made more precise later. The condition for existence and uniqueness of the solution $(\boldsymbol{v}, \psi)$ is given in Section 3.2. With the same notation as in [16] we introduce the operator $\Lambda$ :

$$
\left(\boldsymbol{u}^{0}, \boldsymbol{u}^{1}\right) \in \mathcal{D}(\Omega) \times \mathcal{D}(\Omega) \rightarrow \Lambda\left(\boldsymbol{u}^{0}, \boldsymbol{u}^{1}\right)=\left(\boldsymbol{v}^{\prime}(0),-\boldsymbol{v}(0)\right)
$$

We note that if we can solve the equation

$$
\Lambda\left(\boldsymbol{u}^{0}, \boldsymbol{u}^{1}\right)=\left(\boldsymbol{y}^{1},-\boldsymbol{y}^{0}\right)
$$

then the boundary conditions of problem (9) needed to control the system are

$$
\overline{\boldsymbol{y}}=\overline{\boldsymbol{v}}(\boldsymbol{u}, \varphi), \bar{\theta}=\bar{\psi}(\boldsymbol{u}, \varphi)
$$

Thus the problem we face now is to explicit these boundary conditions and to examine under which conditions equation (11) can be solved. Hence, let $(\boldsymbol{z}, \zeta)$ be the solution to the direct homogeneous problem associated to initial conditions $\left(\boldsymbol{z}^{0}, \boldsymbol{z}^{1}\right) \in \mathcal{D}(\Omega) \times \mathcal{D}(\Omega)$

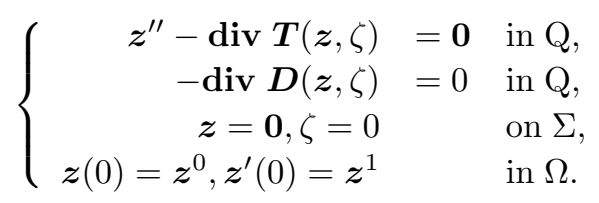

A simple computation yields

$$
\begin{aligned}
\int_{\Omega} \Lambda\left(\boldsymbol{u}^{0}, \boldsymbol{u}^{1}\right) \cdot\left(\boldsymbol{z}^{0}, \boldsymbol{z}^{1}\right)=\int_{\Sigma} c^{i j k l} s_{k l}(\boldsymbol{z}) \bar{v}_{j}(\boldsymbol{u}, \varphi) n_{i} & +e^{i k l} s_{k l}(\boldsymbol{z}) \bar{\psi}(\boldsymbol{u}, \varphi) n_{i} \\
& +e^{k i j} \partial_{k} \zeta \bar{v}_{j}(\boldsymbol{u}, \varphi) n_{i}-d^{i j} \partial_{j} \zeta \bar{\psi}(\varphi) n_{i} .
\end{aligned}
$$

The aim of next section is to show that there exists a choice of boundary values $\overline{\boldsymbol{v}}, \bar{\psi}$ and functional spaces $\boldsymbol{F}$ and $\boldsymbol{F}^{\prime}$ such that operator $\Lambda$ can be uniquely continued from $\boldsymbol{F}$ to $\boldsymbol{F}^{\prime}$, and therefore that the bilinear form

$$
\left(\boldsymbol{u}^{0}, \boldsymbol{u}^{1}\right) \in \boldsymbol{F},\left(\boldsymbol{z}^{0}, \boldsymbol{z}^{1}\right) \in \boldsymbol{F} \longrightarrow \int_{\Omega} \Lambda\left(\boldsymbol{u}^{0}, \boldsymbol{u}^{1}\right) \cdot\left(\boldsymbol{z}^{0}, \boldsymbol{z}^{1}\right)
$$

is continuous and coercive so that equation (11) can be solved by Lax-Milgram theorem.

\subsection{Choice of boundary conditions}

- $(i)$ Case of a sphere $\Omega$.

Let us choose

$$
\left\{\begin{array}{lll}
\overline{\boldsymbol{v}}(\boldsymbol{u}, \varphi) & =\partial_{\boldsymbol{n}} \boldsymbol{u} & \text { on } \Sigma \\
\bar{\psi}(\boldsymbol{u}, \varphi) & =\partial_{\boldsymbol{n}} \varphi & \text { on } \Sigma
\end{array}\right.
$$


This yields

$$
\left\{\begin{aligned}
\int_{\Omega} \Lambda\left(\boldsymbol{u}^{0}, \boldsymbol{u}^{1}\right) \cdot\left(\boldsymbol{z}^{0}, \boldsymbol{z}^{1}\right) & =\int_{\Omega} \Lambda\left(\boldsymbol{z}^{0}, \boldsymbol{z}^{1}\right) \cdot\left(\boldsymbol{u}^{0}, \boldsymbol{u}^{1}\right)=\int_{\Sigma} c(\boldsymbol{u}, \boldsymbol{z})+e(\boldsymbol{z}, \varphi)+e(\boldsymbol{u}, \zeta)-d(\zeta, \varphi), \\
\int_{\Omega} \Lambda\left(\boldsymbol{u}^{0}, \boldsymbol{u}^{1}\right) \cdot\left(\boldsymbol{u}^{0}, \boldsymbol{u}^{1}\right) & =\int_{\Sigma} c(\boldsymbol{u}, \boldsymbol{u})+2 e(\boldsymbol{u}, \varphi)-d(\varphi, \varphi)=\frac{2}{R} E^{0}\left(T-T^{0}\right) .
\end{aligned}\right.
$$

The bilinear operator $\Lambda$ defines a scalar product but not a semi-norm over $\mathcal{D}(\Omega) \times \mathcal{D}(\Omega)$, however the choice of boundary conditions presented below is more relevant for this geometry.

\section{- (ii) Case of a star shaped domain $\Omega$.}

Let us choose

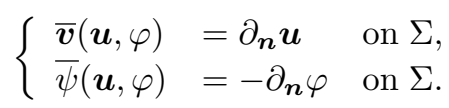

This yields

$$
\left\{\begin{aligned}
\int_{\Omega} \Lambda\left(\boldsymbol{u}^{0}, \boldsymbol{u}^{1}\right) \cdot\left(\boldsymbol{z}^{0}, \boldsymbol{z}^{1}\right) & =\int_{\Sigma} c(\boldsymbol{u}, \boldsymbol{z})-e(\boldsymbol{z}, \varphi)+e(\boldsymbol{u}, \zeta)+d(\zeta, \varphi) \\
\int_{\Omega} \Lambda\left(\boldsymbol{u}^{0}, \boldsymbol{u}^{1}\right) \cdot\left(\boldsymbol{u}^{0}, \boldsymbol{u}^{1}\right) & =\int_{\Sigma} c(\boldsymbol{u}, \boldsymbol{u})+d(\varphi, \varphi)
\end{aligned}\right.
$$

which, according to Corollary (ii), implies

$$
\int_{\Omega} \Lambda\left(\boldsymbol{u}^{0}, \boldsymbol{u}^{1}\right) \cdot\left(\boldsymbol{u}^{0}, \boldsymbol{u}^{1}\right) \geq \int_{\Sigma} c(\boldsymbol{u}, \boldsymbol{u}) \geq \frac{E^{0}}{K R}\left(T-T^{0}\right) .
$$

Hence for strong solution with traces $c(\boldsymbol{u}, \boldsymbol{u}) \in L^{1}(\Sigma), d(\varphi, \varphi) \in L^{1}(\Sigma)$, the semi-norm

$$
\left\|\left(\boldsymbol{u}^{0}, \boldsymbol{u}^{1}\right)\right\|=\left(\int_{\Sigma} c(\boldsymbol{u}, \boldsymbol{u})+d(\varphi, \varphi)\right)^{\frac{1}{2}}
$$

is a norm over $\mathcal{D}(\Omega) \times \mathcal{D}(\Omega)$. Let us note $\boldsymbol{F}$ the closure of $\mathcal{D}(\Omega) \times \mathcal{D}(\Omega)$ for this norm and $\boldsymbol{F}^{\prime}$ its dual space (without identification) (see Komornik $[13,14]$ )

$$
\boldsymbol{F} \subset\left(\boldsymbol{H}^{2}(\Omega) \cap \boldsymbol{H}_{0}^{1}(\Omega)\right) \times \boldsymbol{H}_{0}^{1}(\Omega) .
$$

The operator $\Lambda$ can be uniquely continued in an operator $\Lambda: \boldsymbol{F} \rightarrow \boldsymbol{F}^{\prime}$. The coercivity of $\Lambda$ implies that, for any $\left(\boldsymbol{y}^{1},-\boldsymbol{y}^{0}\right) \in \boldsymbol{F}^{\prime}$, equation (11) has a unique solution $\left(\boldsymbol{u}^{0}, \boldsymbol{u}^{1}\right) \in \boldsymbol{F}$ which implies $c(\boldsymbol{u}, \boldsymbol{u}) \in L^{1}(\Sigma)$ and $d(\varphi, \varphi) \in L^{1}(\Sigma)$, in other words $\partial_{\boldsymbol{n}} \boldsymbol{u} \in \boldsymbol{L}^{2}(\Sigma)$ and $\partial_{\boldsymbol{n}} \varphi \in \boldsymbol{L}^{2}(\Sigma)$.

- (iii) Case of a partition of the boundary $\Gamma$.

We consider the partition of the boundary $\Gamma=\Gamma^{+} \cup \Gamma^{-}, \Gamma^{+} \cap \Gamma^{-}=\emptyset$. Let us choose

$$
\left\{\begin{array}{lllll}
\overline{\boldsymbol{v}}(\boldsymbol{u})=\partial_{\boldsymbol{n}} \boldsymbol{u} & \text { on } \Sigma^{+}, & \overline{\boldsymbol{v}}(\boldsymbol{u})=\mathbf{0} & \text { on } \Sigma^{-} \\
\bar{\psi}(\varphi)=-\partial_{\boldsymbol{n}} \varphi & \text { on } \Sigma^{-}, & \bar{\psi}(\varphi)=0 & \text { on } \Sigma^{+}
\end{array}\right.
$$

This yields

$$
\left\{\begin{aligned}
\int_{\Omega} \Lambda\left(\boldsymbol{u}^{0}, \boldsymbol{u}^{1}\right) \cdot\left(\boldsymbol{z}^{0}, \boldsymbol{z}^{1}\right)= & \int_{\Sigma^{+}} c(\boldsymbol{u}, \boldsymbol{z})+e(\boldsymbol{u}, \zeta)+\int_{\Sigma^{-}} d(\zeta, \varphi)-e(\boldsymbol{z}, \varphi), \\
\int_{\Omega} \Lambda\left(\boldsymbol{u}^{0}, \boldsymbol{u}^{1}\right) \cdot\left(\boldsymbol{u}^{0}, \boldsymbol{u}^{1}\right)= & \int_{\Sigma^{+}} c(\boldsymbol{u}, \boldsymbol{u})+e(\boldsymbol{u}, \varphi)+\int_{\Sigma^{-}}-e(\boldsymbol{u}, \varphi)+d(\varphi, \varphi) \\
& \geq K\left(\int_{\Sigma^{+}} c(\boldsymbol{u}, \boldsymbol{u})-d(\varphi, \varphi)+\int_{\Sigma^{-}}-c(\boldsymbol{u}, \boldsymbol{u})+d(\varphi, \varphi)\right) .
\end{aligned}\right.
$$

The bilinear operator $\Lambda$ does not define a semi-norm over $\mathcal{D}(\Omega) \times \mathcal{D}(\Omega)$. 


\subsection{The controllability result}

TheOREm 5.1 Let $\Omega$ be $x^{0}$-star-shaped with $C^{2}$ boundary and $\boldsymbol{F}$ the Hilbert space defined in 5.2 (ii). Let $\left(\boldsymbol{y}^{1},-\boldsymbol{y}^{0}\right) \in \boldsymbol{F}^{\prime}$ be the initial conditions for problem (9). If the evolution problem (4) associated to initial conditions $\left(\boldsymbol{u}^{0}, \boldsymbol{u}^{1}\right) \in \boldsymbol{F}$ given by (11) has a strong solution $(\boldsymbol{u}, \varphi)$ then there exists a minimum time $T^{0}>0$ and a control $(\overline{\boldsymbol{y}}, \bar{\theta}) \in \boldsymbol{L}^{2}(\Sigma) \times L^{2}(\Sigma)$ acting on the whole boundary $\Sigma$

$$
\overline{\boldsymbol{y}}=\partial_{\boldsymbol{n}} \boldsymbol{u}, \bar{\theta}=-\partial_{\boldsymbol{n}} \varphi \quad \text { on } \Sigma .
$$

This control drives system (9) to rest for $T \geq T^{0}$.

Proof of Theorem 5.1- The existence and uniqueness of the solution $(\boldsymbol{y}, \theta)$ is a consequence of the definition of a weak solution to the nonhomogeneous problem as explained in section 3.2 and the controllability is a direct consequence of the appropriate choice of boundary conditions of $5.2(i i)$.

\section{$6 \quad$ Numerical experiments}

We now present some simple numerical experiments in order to support the result of controllability. For simplicity, we consider the (star-shaped and non Lipschitz domain) unit square $\Omega=(0,1)^{2}$. Following [9], the control $(\overline{\boldsymbol{y}}, \bar{\theta})$ is obtained by minimizing over $\boldsymbol{V} \times \boldsymbol{H}$ the functional $\mathcal{J}: \boldsymbol{V} \times \boldsymbol{H} \rightarrow \mathbb{R}$ defined by

$$
\mathcal{J}\left(\boldsymbol{u}^{\mathbf{0}}, \boldsymbol{u}^{\mathbf{1}}\right)=\frac{1}{2} \int_{\Omega}\left(\Lambda\left(\boldsymbol{u}^{\mathbf{0}}, \boldsymbol{u}^{\mathbf{1}}\right),\left(\boldsymbol{u}^{\mathbf{0}}, \boldsymbol{u}^{\mathbf{1}}\right)\right) d x-<\left(\boldsymbol{u}^{\mathbf{0}}, \boldsymbol{u}^{\mathbf{1}}\right),\left(\boldsymbol{y}^{\mathbf{1}}, \boldsymbol{y}^{\mathbf{0}}\right)>_{\boldsymbol{V} \times \boldsymbol{H}, \boldsymbol{V}^{\prime} \times \boldsymbol{H}^{\prime}} .
$$

This functional, associated to the linear equation (11) is convex, continuous and, from the observability inequality (12), coercive. The minimization of $\mathcal{J}$ is performed using the conjugate gradient (CG) algorithm and requires at each iteration the resolution of the backward and forward system (10). The spatial approximation is done using mass lumping technic (see [5]) and continuous finite elements of order one approximating the spaces $L^{2}(\Omega)$ and $H^{1}(\Omega)$ by the following finite dimensional space:

$$
V_{h}=\left\{v_{h}, v_{h} \in C^{0}(\bar{\Omega}),\left.v_{h}\right|_{Q} \in P_{1}(Q), \forall Q \in Q_{h}\right\},
$$

where $P_{1}(Q)$ denotes the space of polynomial functions of degree $\leq 1$ on $Q$, the notation $\left(Q_{h}\right)_{h>0}$ stands for a regular quasi-uniform family of quadrangulations characterized by the space step $h$ such that $\bar{\Omega}=\cup_{Q \in Q_{h}} Q$. Remark that such approximation is non-conforming for our problem, since the space $\boldsymbol{F}$ (defined in Section $5.2 \mathrm{ii}$ )) would require at least $C^{1}$-finite element. The temporal approximation is performed in a standard way using centered finite differences of order two. Moreover, as is well known since [9], such approximation may not provide convergent results with respect to the parameters of discretization, when the initial data $\left(\boldsymbol{y}^{\mathbf{0}}, \boldsymbol{y}^{\mathbf{1}}\right)$ are generated by high frequency modes: in that case, the approximation $\mathcal{J}_{h}$ of $\mathcal{J}$ is not uniformly coercive with respect to $h$ (we also refer to $[4,20]$ ). In order to avoid this phenomenon and obtain a convergent sequence of controls with respect to $h$, we consider only regular initial data. Moreover, we use the Bi-Grid method (introduced in [9] in the context of the wave equation and apply in [2] for the elasticity system), which consists to compute, at each iteration of the CG algorithm, the new descent direction on a coarse grid. This method permits to improve the numerical stability. We refer to $[4,20]$ for others recent remedies in a similar context in the critical situation where the initial position $\boldsymbol{y}^{\mathbf{0}}$ is discontinuous. The 
algorithm, initialized by $\left(\boldsymbol{u}^{\mathbf{0}}, \boldsymbol{u}^{\mathbf{1}}\right)=(\mathbf{0}, \mathbf{0})$ is stopped as soon as the residual $r^{(k)}$ (related to the norm of the descent direction) of the algorithm becomes lower than $10^{-8}$. Finally, in order to have a smooth control at time $t=0$ and $t=T$, we introduce a cut-off function $\rho \in V_{\eta}=\left\{v, v \in C^{1}([0, T]), v(0)=v(T)=v^{\prime}(0)=v^{\prime}(T)=0, v>0 \in\right] \eta, T-\eta[\}$ for any $0<\eta<<T$ and search for a control of (9) under the form $(\rho \overline{\boldsymbol{y}}, \rho \theta) \in\left(L^{2}(\Sigma)\right)^{3}$. This is natural from a mechanical point of view (the control can not suddenly acts at time 0 ) and improves the performances of the CG algorithm.

For the numerical simulations we have selected the PZT4 material which is of particular interest in the design of transformers, its characteristics (mass density $\rho$, stiffness $c_{\alpha \beta}$, piezoelectric coupling $e_{\alpha \alpha}$ and permittivity $\varepsilon_{11}$ ) are given in Table 1 (see [8]).

\begin{tabular}{c|c|c|c|c|c}
$c_{11}\left(\mathrm{~N} / \mathrm{m}^{2}\right)$ & $c_{12}\left(\mathrm{~N} / \mathrm{m}^{2}\right)$ & $c_{22}\left(\mathrm{~N} / \mathrm{m}^{2}\right)$ & $\rho\left(\mathrm{Kg} / \mathrm{m}^{3}\right)$ & $e_{11}=e_{22}\left(\mathrm{C} / \mathrm{m}^{2}\right)$ & $\varepsilon_{11}(\mathrm{~F} / \mathrm{m})$ \\
\hline $13.9 \times 10^{10}$ & $7.8 \times 10^{10}$ & $2.36 \times 10^{10}$ & $7.5 \times 10^{3}$ & 12.7 & $65 \times 10^{-10}$
\end{tabular}

Table 1: Constants of the PZT4 material.

In the next sections, some numerical experiments obtained for different positions of the control $\left(\bar{y}_{1}, \bar{y}_{2}, \bar{\theta}\right)$ for the piezo-electric system (9) are discussed. The notation $S\left(\bar{y}_{1}, \bar{y}_{2}, \theta\right)=$ $\left(\Sigma_{1}, \Sigma_{2}, \Sigma_{3}\right)$ used in the sequel signifies that the support of $\bar{y}_{1}, \bar{y}_{2}$ and $\bar{\theta}$ are $\Sigma_{1}, \Sigma_{2}$ and $\Sigma_{3}$ respectively (for any $\Sigma_{i} \subset \Sigma, i=1 . .3$ ). In particular, we consider the supports $\Sigma^{ \pm}=$ $\Gamma^{ \pm} \times(0, T)$ with

$$
\Gamma^{+}=\left\{\boldsymbol{x}=\left(x_{1}, x_{2}\right) \in \Gamma, x_{2}\left(1-x_{1}\right)=0\right\}, \quad \Gamma^{-}=\left\{\boldsymbol{x}=\left(x_{1}, x_{2}\right) \in \Gamma, x_{1}\left(1-x_{2}\right)=0\right\} .
$$

The space step and time step parameter are taken equal to $h=1 / 60$ and $\Delta t=h / 4$ respectively. Finally, we use $\eta=T / 10$.

\subsection{Example 1}

We use the simplest low frequency mode as initial condition on the unit square :

$$
\boldsymbol{y}^{\mathbf{0}}=\left(\sin \left(\pi x_{1}\right) \sin \left(\pi x_{2}\right), 0\right), \quad \boldsymbol{y}^{\mathbf{1}}=(0,0), \quad\left(x_{1}, x_{2}\right) \in(0,1)^{2}
$$

and take $T=4$.

Let us first consider the situation of the theoretical part (precisely of Section $5.2 \mathrm{ii}$ )) where the system (9) is controlled by an elastic and electric Dirichlet force on the whole boundary $\Sigma$ (i.e. $S\left(\bar{y}_{1}, \bar{y}_{2}, \bar{\theta}\right)=(\Sigma, \Sigma, \Sigma)$ ). Figure 1-left depicts the evolution of the total energy and kinetic energy with respect to the time $t$ in $[0, T]$ highlighting the vibrations of the structure. We obtain at the convergence the ratio $E(T) / E(0) \approx 7.58 \times 10^{-5}(E(t)$ denotes (the approximation of) the energy of the controlled system (9) at time $t$ ) which illustrates the (numerical) exact controllability. On the other hand, Figure 1-right depicts the evolution with respect to the time of the $L^{2}(\Gamma)$-norm of the elastic control $\bar{y}_{1}, \bar{y}_{2}$ and electric control $\bar{\theta}$. From Table 2, we observe that the corresponding $L^{2}(\Sigma)$-norm of the three control are similar. Finally, the convergence obtained after only 7 iterations is very fast (with respect to the value of $h$ ). Numerical values are collected in Table 2 .

Following the discussion of Section 5.2, we then analyze to which extend we may reduced the support of the control. Figure 2 and Table 3 address the situation $S\left(\bar{y}_{1}, \bar{y}_{2}, \bar{\theta}\right)=$ $\left(\Sigma^{+}, \Sigma^{+}, \Sigma^{+}\right)$still yielding a positive answer (of controllability). Numerical values are very similar : the norm of the controls and the number of iterations are slightly greater. Remark 

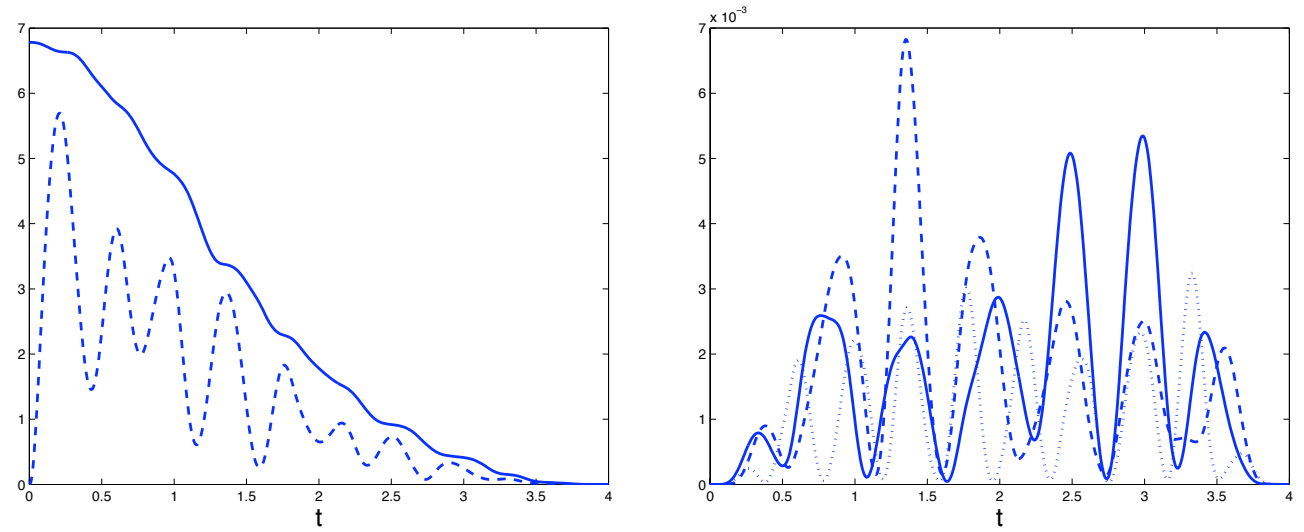

Figure 1: $S\left(\bar{y}_{1}, \bar{y}_{2}, \bar{\theta}\right)=(\Sigma, \Sigma, \Sigma)$ - Left: Total energy (solid line) and kinetic energy (dashed line) vs. $t \in[0, T]$; Right: $L^{2}(\Gamma)$-norm of the control $\bar{y}_{1}$ (solid line), $\bar{y}_{2}$ (dashed line) and $\bar{\theta}$ (dotted line) vs. $t \in[0, T]$ (Example 1$)$.

\begin{tabular}{c|c|c|c|c|c|c}
$\left\|\overline{y_{1}}\right\|_{L^{2}(\Sigma)}$ & $\left\|\overline{y_{2}}\right\|_{L^{2}(\Sigma)}$ & $\|\bar{\theta}\|_{L^{2}(\Sigma)}$ & $E(T) / E(0)$ & $\|\boldsymbol{y}(T)\|_{L^{2}(\Gamma)}$ & $\left\|\boldsymbol{y}^{\prime}(T)\right\|_{L^{2}(\Gamma)}$ & Nb. It \\
\hline $7.87 \times 10^{-2}$ & $7.86 \times 10^{-2}$ & $6.29 \times 10^{-2}$ & $7.58 \times 10^{-5}$ & $2.19 \times 10^{-4}$ & $1.55 \times 10^{-2}$ & 7
\end{tabular}

Table 2: Numerical values corresponding to $S\left(\bar{y}_{1}, \bar{y}_{2}, \bar{\theta}\right)=(\Sigma, \Sigma, \Sigma)$ (Example 1$)$.
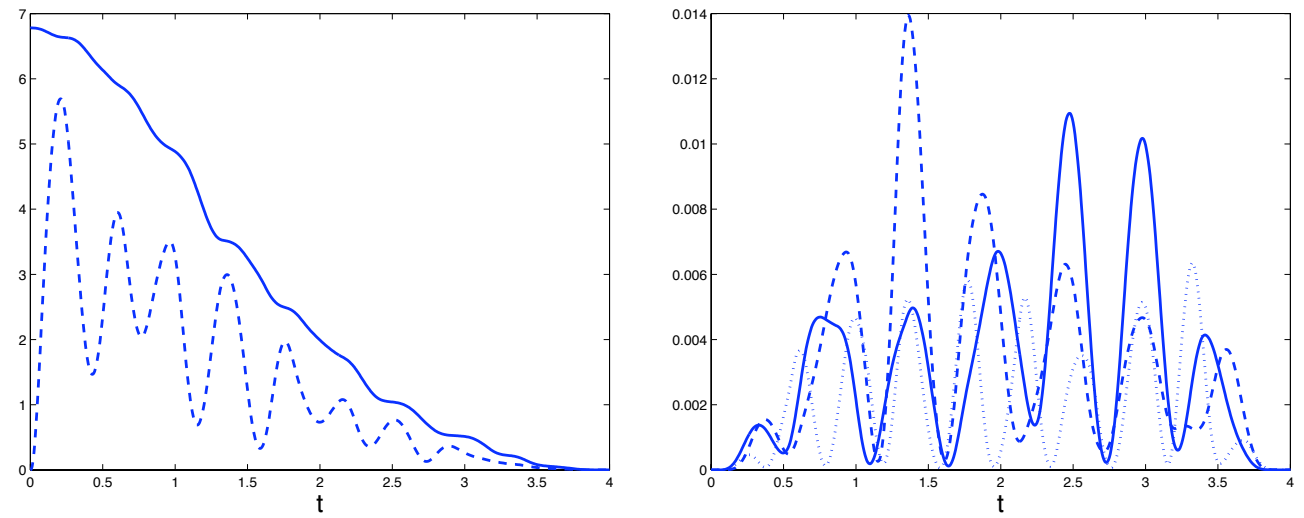

Figure 2: $S\left(\bar{y}_{1}, \bar{y}_{2}, \bar{\theta}\right)=\left(\Sigma^{+}, \Sigma^{+}, \Sigma^{+}\right)$- Left: Total energy (solid line) and kinetic energy (dashed line) vs. $t \in[0, T]$; Right: $L^{2}(\Gamma)$ - norm of the control $\bar{y}_{1}$ (solid line), $\bar{y}_{2}$ (dashed line) and $\bar{\theta}$ (dotted line) vs. $t \in[0, T]$ (Example 1$)$.

\begin{tabular}{c|c|c|c|c|c|c}
$\left\|\overline{y_{1}}\right\|_{L^{2}(\Sigma)}$ & $\left\|\overline{y_{2}}\right\|_{L^{2}(\Sigma)}$ & $\|\bar{\theta}\|_{L^{2}(\Sigma)}$ & $E(T) / E(0)$ & $\|\boldsymbol{y}(T)\|_{L^{2}(\Gamma)}$ & $\left\|\boldsymbol{y}^{\prime}(T)\right\|_{L^{2}(\Gamma)}$ & Nb. It \\
\hline $1.11 \times 10^{-1}$ & $1.12 \times 10^{-1}$ & $8.90 \times 10^{-2}$ & $2.40 \times 10^{-4}$ & $3.76 \times 10^{-4}$ & $2.86 \times 10^{-2}$ & 9
\end{tabular}

Table 3: Numerical values corresponding to $S\left(\bar{y}_{1}, \bar{y}_{2}, \bar{\theta}\right)=\left(\Sigma^{+}, \Sigma^{+}, \Sigma^{+}\right)$(Example 1). 
that this observation, obtained for one single mode, is not in contradiction with Section 5.2.

Further numerical simulations then suggest that the controllability still holds if the support is less restrictive. For instance, the cases $S\left(\bar{y}_{1}, \bar{y}_{2}, \bar{\theta}\right)=\left(\Sigma^{+}, \Sigma^{+}, \Sigma^{-}\right)$(Table 4$)$, $S\left(\bar{y}_{1}, \bar{y}_{2}, \bar{\theta}\right)=(\Sigma, \Sigma, \emptyset)$ (Table 5 ) for which the electric control is simply removed also leads to controllability. On the contrary, considering the case $S\left(\bar{y}_{1}, \bar{y}_{2}, \bar{\theta}\right)=(\emptyset, \emptyset, \Sigma)$, we observe that the electric control $\bar{\theta}$ is not sufficient to drive to rest the field $\boldsymbol{y}$ and $\theta$. Observe that the case $S\left(\bar{y}_{1}, \bar{y}_{2}, \bar{\theta}\right)=\left(\Sigma^{+}, \Sigma^{+}, \Sigma^{-}\right)$produces very similar numerical values than the case $S\left(\bar{y}_{1}, \bar{y}_{2}, \bar{\theta}\right)=\left(\Sigma^{+}, \Sigma^{+}, \Sigma^{+}\right)$.
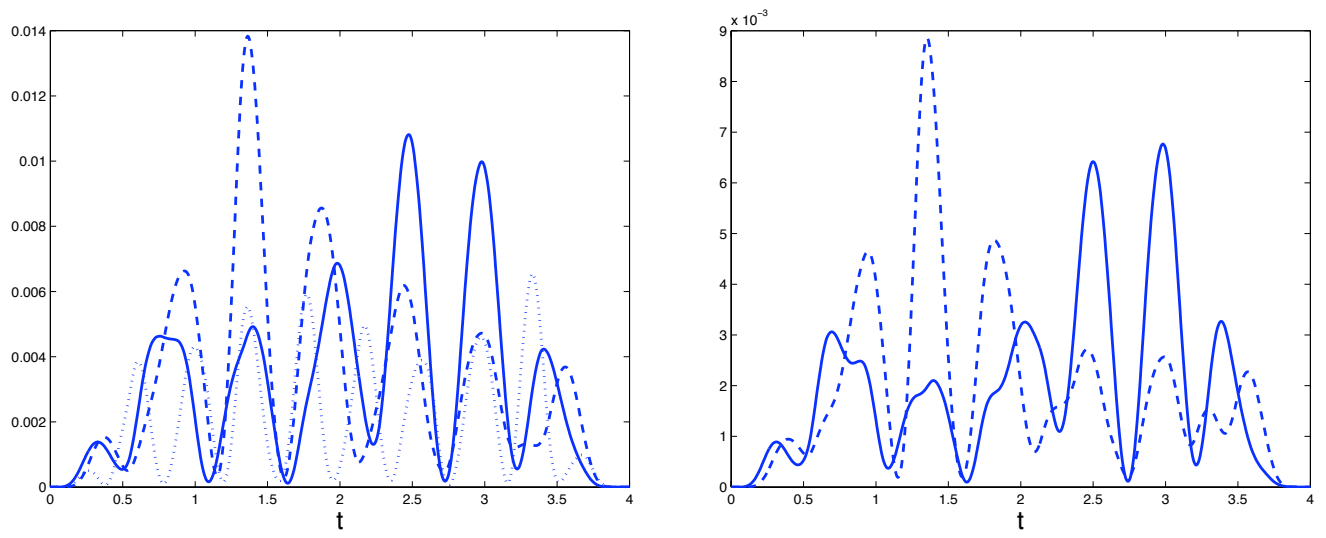

Figure 3: $L^{2}(\Gamma)$-norm of the controls in the case $S\left(\bar{y}_{1}, \bar{y}_{2}, \bar{\theta}\right)=\left(\Sigma^{+}, \Sigma^{+}, \Sigma^{-}\right)($Left $)$and $S\left(\bar{y}_{1}, \bar{y}_{2}, \bar{\theta}\right)=(\Sigma, \Sigma, \emptyset)$ (Right) vs. $t \in[0, T]$ (Example 1$)$.

\begin{tabular}{c|c|c|c|c|c|c}
$\left\|\overline{y_{1}}\right\|_{L^{2}(\Sigma)}$ & $\left\|\overline{y_{2}}\right\|_{L^{2}(\Sigma)}$ & $\|\bar{\theta}\|_{L^{2}(\Sigma)}$ & $E_{h}(T) / E_{h}(0)$ & $\|\boldsymbol{y}(T)\|_{L^{2}(\Gamma)}$ & $\left\|\boldsymbol{y}^{\prime}(T)\right\|_{L^{2}(\Gamma)}$ & $\mathrm{Nb} . \mathrm{It}$ \\
\hline $1.11 \times 10^{-1}$ & $1.12 \times 10^{-1}$ & $8.94 \times 10^{-2}$ & $1.47 \times 10^{-4}$ & $3.05 \times 10^{-4}$ & $2.26 \times 10^{-2}$ & 8
\end{tabular}

Table 4: Numerical values corresponding to $S\left(\bar{y}_{1}, \bar{y}_{2}, \bar{\theta}\right)=\left(\Sigma^{+}, \Sigma^{+}, \Sigma^{-}\right)$(Example 1).

\begin{tabular}{c|c|c|c|c|c|c}
$\left\|\overline{y_{1}}\right\|_{L^{2}(\Sigma)}$ & $\left\|\overline{y_{2}}\right\|_{L^{2}(\Sigma)}$ & $\|\bar{\theta}\|_{L^{2}(\Sigma)}$ & $E_{h}(T) / E_{h}(0)$ & $\|\boldsymbol{y}(T)\|_{L^{2}(\Gamma)}$ & $\left\|\boldsymbol{y}^{\prime}(T)\right\|_{L^{2}(\Gamma)}$ & $\mathrm{Nb} . \mathrm{It}$ \\
\hline $8.69 \times 10^{-2}$ & $8.68 \times 10^{-2}$ & 0. & $6.23 \times 10^{-5}$ & $1.82 \times 10^{-4}$ & $1.27 \times 10^{-2}$ & 6
\end{tabular}

Table 5: Numerical values corresponding to $S\left(\bar{y}_{1}, \bar{y}_{2}, \bar{\theta}\right)=(\Sigma, \Sigma, \emptyset)$ (Example 1$)$.

We still observe controllability if the elastic controls are supported on $\Sigma^{+}$(i.e. $S\left(\bar{y}_{1}, \bar{y}_{2}, \bar{\theta}\right)=$ $\left(\Sigma^{+}, \Sigma^{+}, \emptyset\right)$ ) (see Table 6) and if one of $\bar{y}_{1}$ or $\bar{y}_{2}$ is supported on the whole boundary (i.e. $S\left(\bar{y}_{1}, \bar{y}_{2}, \bar{\theta}\right)=(\Sigma, \emptyset, \emptyset)$ ) (see Table 7 ). The controllability, lost if $\bar{y}_{1}$ (or $\bar{y}_{2}$ ) only acts on $\Sigma^{+}$, is recovered if we add for instance the electric control $\bar{\theta}$ on $\Sigma^{-}$(or on $\Sigma^{+}$) (Figure 5 and Table 8).

\subsection{Example 2}

Still on the unit square, we now consider the following initial data

$$
\boldsymbol{y}^{\mathbf{0}}=\left(x_{1}\left(1-x_{1}\right) x_{2}\left(1-x_{2}\right) e^{-30\left(x_{1}-0.3\right)^{2}-30\left(x_{2}-0.3\right)^{2}}, 0\right), \boldsymbol{y}^{\mathbf{1}}=(0,0)
$$



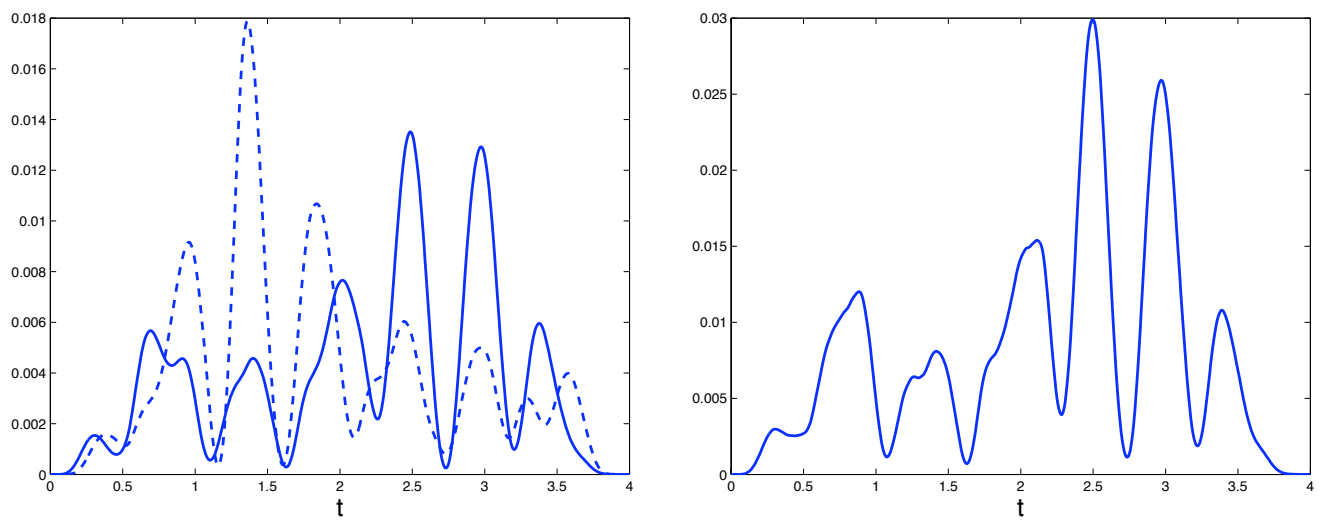

Figure 4: $L^{2}(\Gamma)$-norm of the controls in the case $S\left(\bar{y}_{1}, \bar{y}_{2}, \bar{\theta}\right)=\left(\Sigma^{+}, \Sigma^{+}, \emptyset\right)($ Left $)$ and $S\left(\bar{y}_{1}, \bar{y}_{2}, \bar{\theta}\right)=(\Sigma, \emptyset, \emptyset)$ (Right) vs. $t \in[0, T]$ (Example 1$)$.

\begin{tabular}{c|c|c|c|c|c|c}
$\left\|\overline{y_{1}}\right\|_{L^{2}(\Sigma)}$ & $\left\|\overline{y_{2}}\right\|_{L^{2}(\Sigma)}$ & $\|\bar{\theta}\|_{L^{2}(\Sigma)}$ & $E_{h}(T) / E_{h}(0)$ & $\|\boldsymbol{y}(T)\|_{L^{2}(\Gamma)}$ & $\left\|\boldsymbol{y}^{\prime}(T)\right\|_{L^{2}(\Gamma)}$ & $\mathrm{Nb}$. It \\
\hline $1.22 \times 10^{-1}$ & $1.24 \times 10^{-1}$ & 0. & $1.5 \times 10^{-4}$ & $3.33 \times 10^{-4}$ & $2.28 \times 10^{-2}$ & 7
\end{tabular}

Table 6: Numerical values corresponding to $S\left(\bar{y}_{1}, \bar{y}_{2}, \bar{\theta}\right)=\left(\Sigma^{+}, \Sigma^{+}, \emptyset\right)$ (Example 1).

\begin{tabular}{c|c|c|c|c|c|c}
$\left\|\overline{y_{1}}\right\|_{L^{2}(\Sigma)}$ & $\left\|\overline{y_{2}}\right\|_{L^{2}(\Sigma)}$ & $\|\bar{\theta}\|_{L^{2}(\Sigma)}$ & $E_{h}(T) / E_{h}(0)$ & $\|\boldsymbol{y}(T)\|_{L^{2}(\Gamma)}$ & $\left\|\boldsymbol{y}^{\prime}(T)\right\|_{L^{2}(\Gamma)}$ & $\mathrm{Nb} . \mathrm{It}$ \\
\hline $1.76 \times 10^{-1}$ & 0. & 0. & $8.85 \times 10^{-4}$ & $8.25 \times 10^{-4}$ & $5.66 \times 10^{-2}$ & 15
\end{tabular}

Table 7: Numerical values corresponding to $S\left(\bar{y}_{1}, \bar{y}_{2}, \bar{\theta}\right)=(\Sigma, \emptyset, \emptyset)$ (Example 1).
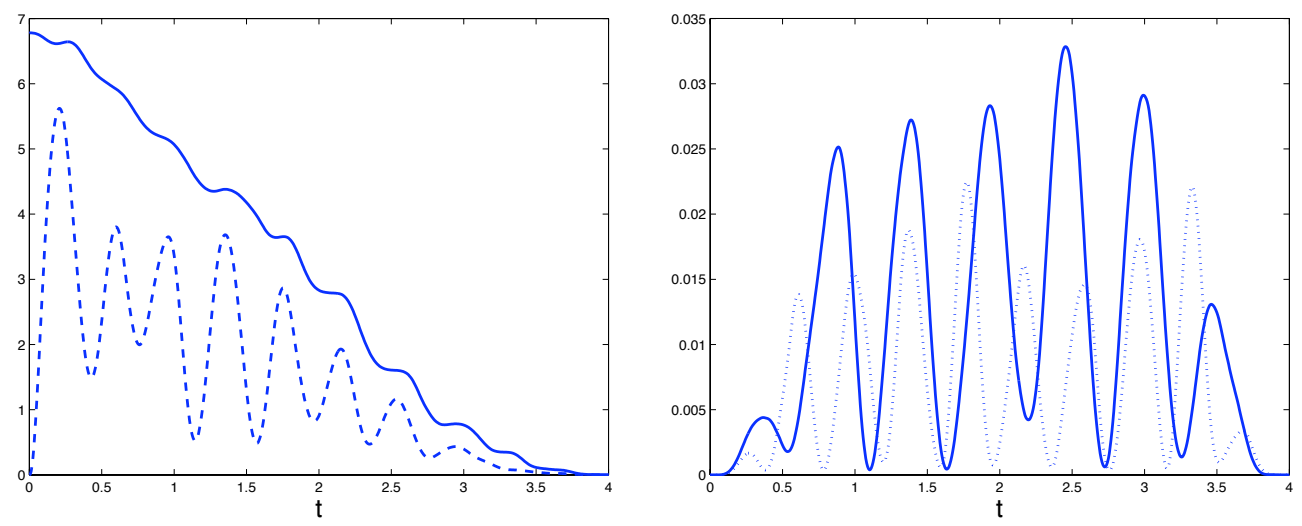

Figure 5: $S\left(\bar{y}_{1}, \bar{y}_{2}, \bar{\theta}\right)=\left(\Sigma^{+}, \emptyset, \Sigma^{-}\right)$- Left: Total energy (solid line) and kinetic energy (dashed line) vs. $t \in[0, T]$; Right: $L^{2}(\Gamma)$ - norm of the control $\bar{y}_{1}$ (solid line) and $\bar{\theta}$ (dotted line) vs. $t \in[0, T]$ (Example 1$)$.

\begin{tabular}{c|c|c|c|c|c|c}
$\left\|\overline{y_{1}}\right\|_{L^{2}(\Sigma)}$ & $\left\|\overline{y_{2}}\right\|_{L^{2}(\Sigma)}$ & $\|\bar{\theta}\|_{L^{2}(\Sigma)}$ & $E_{h}(T) / E_{h}(0)$ & $\|\boldsymbol{y}(T)\|_{L^{2}(\Gamma)}$ & $\left\|\boldsymbol{y}^{\prime}(T)\right\|_{L^{2}(\Gamma)}$ & Nb. It \\
\hline $2.12 \times 10^{-1}$ & 0. & $1.69 \times 10^{-1}$ & $7.56 \times 10^{-4}$ & $8.16 \times 10^{-4}$ & $5.13 \times 10^{-2}$ & 16
\end{tabular}

Table 8: Numerical values corresponding to $S\left(\bar{y}_{1}, \bar{y}_{2}, \bar{\theta}\right)=\left(\Sigma^{+}, \emptyset, \Sigma^{-}\right)$(Example 1). 
in order to exhibit more frequency modes than the first example. The case $S\left(\bar{y}_{1}, \bar{y}_{2}, \bar{\theta}\right)=$ $(\Sigma, \Sigma, \Sigma)$ controllable for $T=4$ is summarized in Figure 6 and Table 9. The evolution in time of the $L^{2}$-norm of the controls are slightly less smooth than for the first example and the number of iteration to convergence is greater. Moreover, our numerical experiment suggests that $T=4$ is not sufficient to control the system from the boundary $\Sigma^{+}$(i.e. $S\left(\bar{y}_{1}, \bar{y}_{2}, \bar{\theta}\right)=$ $\left.\left(\Sigma^{+}, \Sigma^{+}, \Sigma^{+}\right)\right)$. The controllability is recovered if, for instance, $T=5$. Figure 8 gives the electric field $\theta$ in $\Omega$ at time $t=i T / 5, i=0, . ., 5$ for the case $S\left(\bar{y}_{1}, \bar{y}_{2}, \bar{\theta}\right)=\left(\Sigma^{+}, \Sigma^{+}, \Sigma^{-}\right)$for which the controllability holds (see also Figure 7 and Table 10). Remark that the electric field $\theta$ is not equal to zero at time $t=0$.
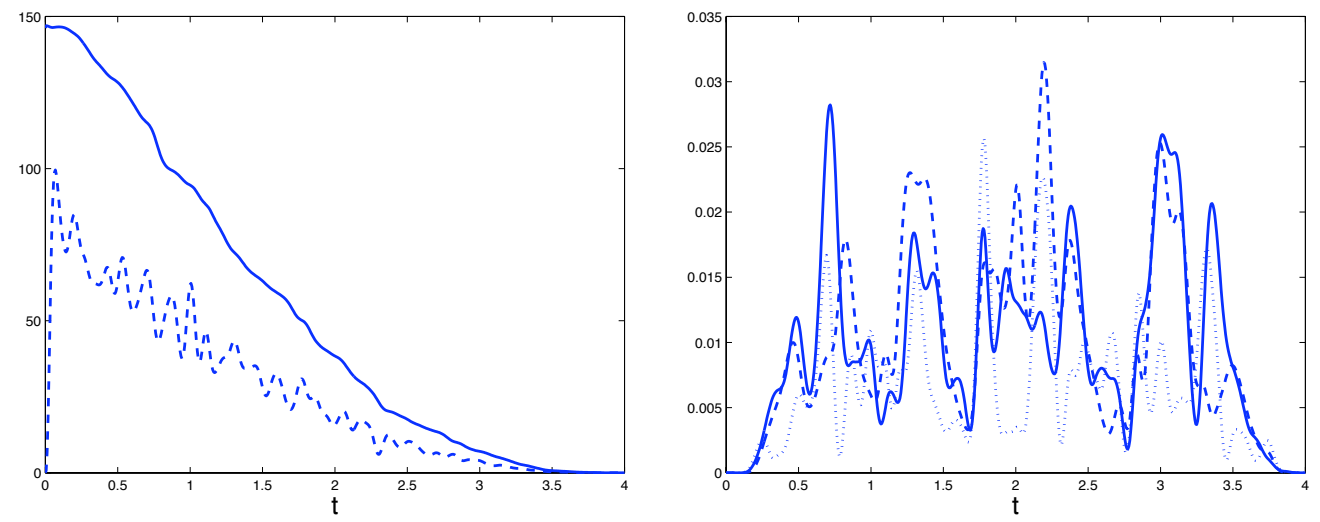

Figure 6: $S\left(\bar{y}_{1}, \bar{y}_{2}, \bar{\theta}\right)=(\Sigma, \Sigma, \Sigma)$ - Left: Total energy (solid line) and kinetic energy (dashed line) vs. $t \in[0, T]$; Right: $L^{2}(\Gamma)$ - norm of the control $\bar{y}_{1}$ (solid line), $\bar{y}_{2}$ (dashed line) and $\bar{\theta}$ (dotted line) vs. $t \in[0, T]$ (Example 2$)$.

\begin{tabular}{c|c|c|c|c|c|c}
$\left\|\overline{y_{1}}\right\|_{L^{2}(\Sigma)}$ & $\left\|\overline{y_{2}}\right\|_{L^{2}(\Sigma)}$ & $\|\bar{\theta}\|_{L^{2}(\Sigma)}$ & $E(T) / E(0)$ & $\|\boldsymbol{y}(T)\|_{L^{2}(\Gamma)}$ & $\left\|\boldsymbol{y}^{\prime}(T)\right\|_{L^{2}(\Gamma)}$ & Nb. It \\
\hline $1.97 \times 10^{-1}$ & $1.99 \times 10^{-1}$ & $1.62 \times 10^{-1}$ & $2.29 \times 10^{-4}$ & $2.10 \times 10^{-3}$ & $1.29 \times 10^{-1}$ & 10
\end{tabular}

Table 9: Numerical values corresponding to $S\left(\bar{y}_{1}, \bar{y}_{2}, \bar{\theta}\right)=(\Sigma, \Sigma, \Sigma)$ (Example 2).

\begin{tabular}{|c|c|c|c|c|c|c|}
\hline & $\left\|\overline{y_{2}}\right\|_{L^{2}(\Sigma)}$ & $\|\bar{\theta}\|_{L^{2}(\Sigma)}$ & $E(T) / E(0)$ & $\boldsymbol{y}(T) \|_{L^{2}(\Gamma)}$ & $\left\|\boldsymbol{y}^{\prime}(T)\right\|_{L^{2}(\Gamma)}$ & $\mathrm{Nb}$. It \\
\hline $2.73 \times$ & $3.01 \times 10^{-1}$ & $2.21 \times 10^{-}$ & $5.02 \times 10^{-4}$ & $3.77 \times 10^{-3}$ & $1.90 \times 10^{-1}$ & 11 \\
\hline
\end{tabular}

Table 10: Numerical values corresponding to $S\left(\bar{y}_{1}, \bar{y}_{2}, \bar{\theta}\right)=\left(\Sigma^{+}, \Sigma^{+}, \Sigma^{-}\right)$(Example 2).

\section{Concluding remarks}

We have proved in this work an exact controllability result for a piezo-electric PDE system, assuming the (Dirichlet) control active on the whole boundary and the time of controllability large enough. The proof is based on the observability inequality through the multiplier method and HUM. Numerical experiments confirm these results and also suggest that the assumption on the support may be relaxed: for instance, the controllability is still observed 

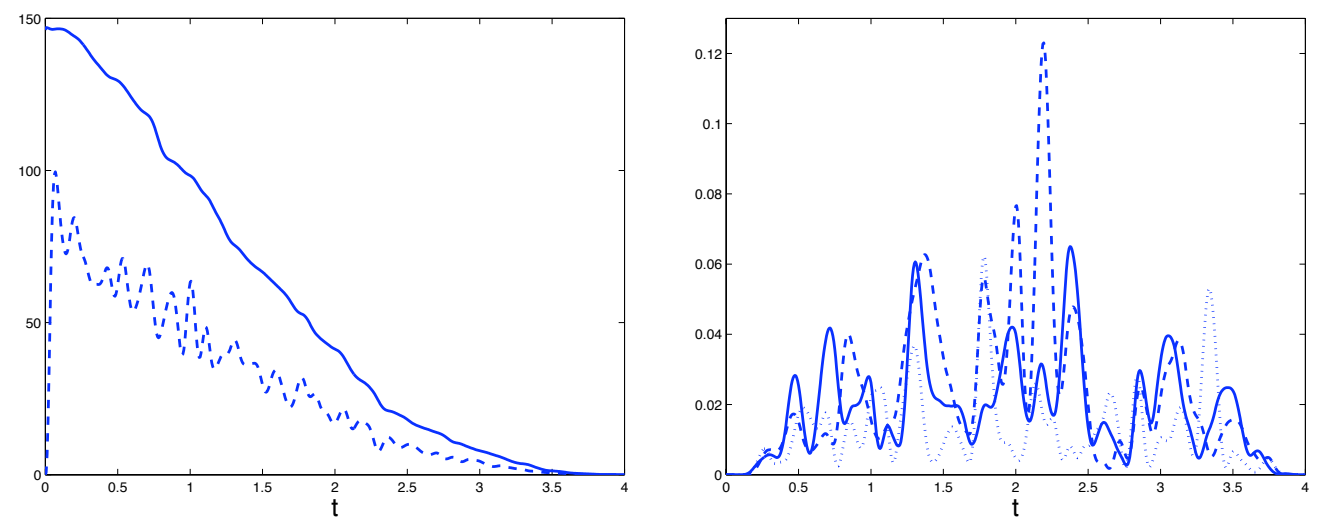

Figure 7: $S\left(\bar{y}_{1}, \bar{y}_{2}, \bar{\theta}\right)=(\Sigma, \Sigma, \Sigma)$ - Left: Total energy (solid line) and kinetic energy (dashed line) vs. $t \in[0, T]$; Right: $L^{2}(\Gamma)$ - norm of the control $\bar{y}_{1}$ (solid line), $\bar{y}_{2}$ (dashed line) and $\bar{\theta}$ (dotted line) vs. $t \in[0, T]$ (Example 2$)$.

when the elastic controls act on the part $\Sigma^{+}$of the boundary while the electric one acts on the complementary part $\Sigma^{-}$. The numerical experiments also suggest that the elastic control suffices to drive to rest both the elastic and electric field. It would be interesting to analyze deeper the interplay between the elastic and electric control, and examine in particular their optimal respective position on the boundary, as it is done in a similar context in [21]. It is of interest also to note that the boundary control has been obtained for so-called shallow shells in [18] (with the condition of shallowness removed in [1]) and to the observability and stabilization of three dimensional bodies in [11, 12] and [19].

Acknowlegdments. The second author thanks Professor Mark Asch (Université de Picardie Jules Verne) for interesting discussions related to this work.

\section{References}

[1] F. Ammar-Khodja, G. Geymonat, A. Münch, On the exact controllability of a system of mixed order with essential spectrum, C.R. Acad. Sci. Paris, Sér. I, 346, 629-634, (2008).

[2] M. Asch, G. Lebeau, Geometrical aspects of exact controllability for the wave equation: a numerical study, ESAIM-COCV, 3, 163-212 (1998).

[3] M. Bernadou, M.C. Delfour, Intrisic asymptotic model of piezoelectric shells, in Optimal Control of Complex Structures, K.-H. Hoffmann, I. Lasiecka, G. Leugering, J. Sprekels, F. Troltzsch (Eds), International Series of Numerical Mathematics, Birkhauser-Verlag, 59-72 (2002).

[4] C. Castro, S. Micu, A. Münch, Numerical approximation of the boundary control for the wave equation with mixed finite elements in a square, IMA Journal of Numerical Analysis, 28(1), 186-214 (2008)

[5] G.C. Cohen, Higher order numerical method for transient wave equation, Scientific computation, Springer, Berlin, 2002. 

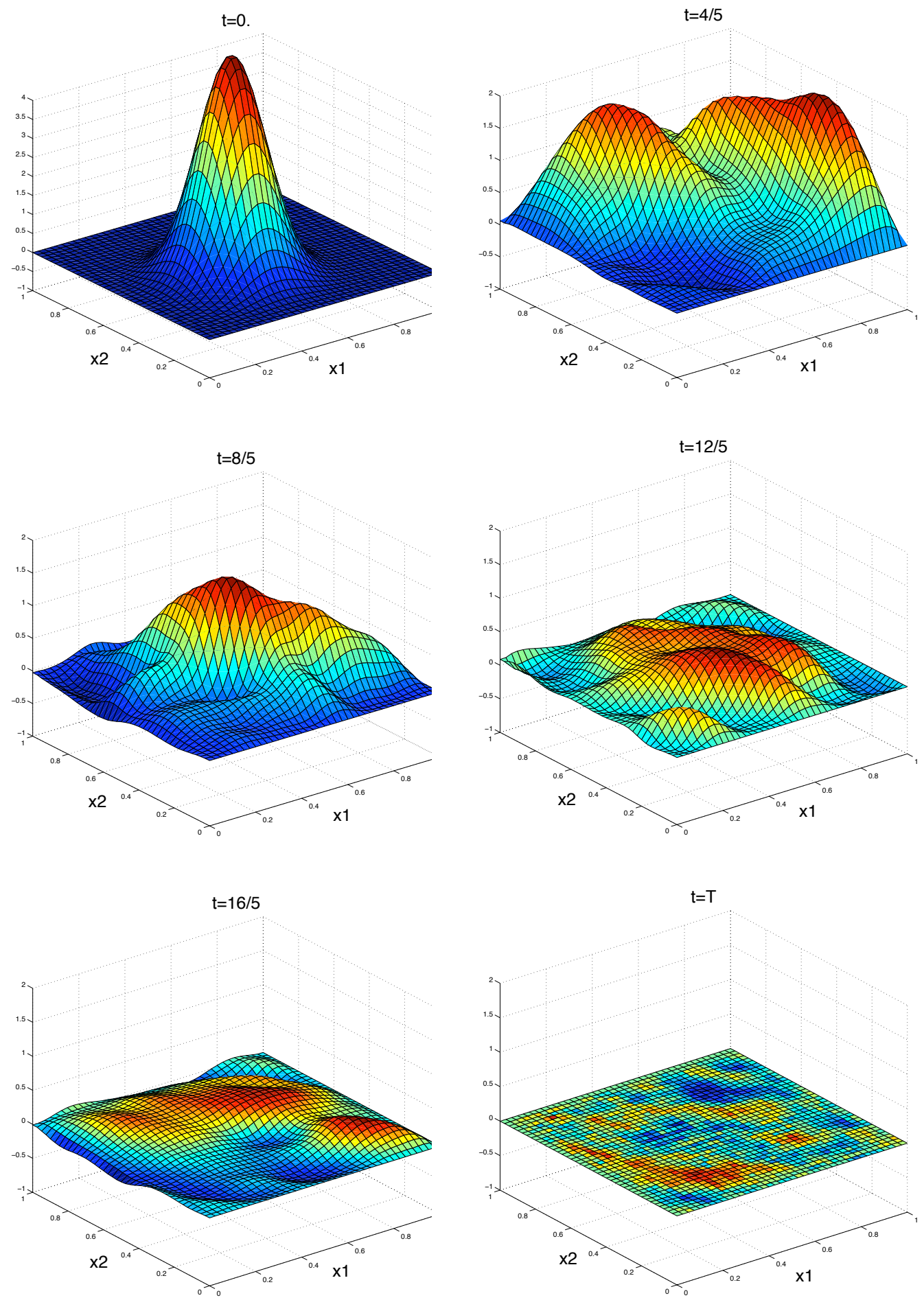

Figure 8: $\quad S\left(\bar{y}_{1}, \bar{y}_{2}, \bar{\theta}\right)=\left(\Sigma^{+}, \Sigma^{+}, \Sigma^{-}\right)-$Electric field $\theta$ in $\Omega$ at time $t=$ $0, T / 5,2 T / 5,3 T / 5,4 T / 5$ and $t=T=4$ (Example 2). 
[6] E. Crépeau, C. Prieur, Control of a clamped-free beam by a piezoelectric actuator. Control. Optimal. Calc. Var., 12, 545-563, (2006).

[7] Ph. Destuynder, A mathematical analysis of a smart-beam which is equipped with piezoelectric actuators. Control Cybernet., 28, 503-530, (1999).

[8] E. Dieulesaint, D. Royer, Ondes élastiques dans les solides, application au traitement du signal. Masson, Paris, 1974.

[9] R. Glowinski, C.H. Li, J.L. Lions, A numerical approach to the exact boundary controllability of the wave equation (I). Dirichlet controls: Description of the numerical methods, Japan J. Appl. Math. 7, 1-76 (1990).

[10] R. Glowinski, Ensuring well-posedness by analogy: Stokes problem and boundary control for the wave equation, J. Comput. Phys, 103, 189-221 (1992).

[11] B. Kapitonov, G. Perla-Menzala and B. Miara, Stabilization of a layered piezoelectric 3-D body by boundary dissipation, ESAIM-COCV, 12(2), 198-221, (2006).

[12] B. Kapitonov, G. Perla-Menzala and B. Miara, Boundary Observation and Exact Control of a Quasi-electrostatic Piezoelectric System in Multilayered Media, SIAM, J. Control and Optimization, 46 (3), 1080-1097, (2007).

[13] V. Komornik, Exact controllability and stabilization. The multiplier method. Masson, 1994.

[14] V. Komornik, Rapid boundary stabilization of Maxwell's equations. Articles dédiés à Jacques-Louis Lions, Gauthiers-Villars, 611-622, (1998).

[15] J.-L. Lions, E.Magenes, Problèmes aux limites non homogènes. Vol 2, Dunod, 1968.

[16] J.-L. Lions, Contrôlabilité exacte, perturbations et stabilisation de systèmes distribués. Tome 1, Masson, 1988.

[17] B. Miara, Contrôlabilité d'un corps piézoélectrique, Comptes Rendus de l'Académie des Sciences Paris, Ser. I, 333, 267-270, (2001).

[18] B. Miara, Exact controllability of piezoelectric shells, Fourth Conference on Elliptic and Parabolic Problems, Gaeta, 434-441, (2002).

[19] B. Miara, M.L. Santos, Stabilization of piezoelectric body, work in progress.

[20] A. Münch, A uniformly controllable and implicit scheme for the 1-D wave equation, Mathematical Modelling and Numerical Analysis, 39(2), 377-418 (2005).

[21] A. Münch, Optimal design of the support of the control for the 2-D wave equation : numerical investigations, Int. J. Numer. Analysis and Modeling, 5(2), 331-351 (2008).

[22] A. Preumont, A. Francois, P. De Man, N. Loix and K. Henrioulle, Distributed sensors with piezoelectric films in design of spatial filters for structural control, Journal of Sound and Vibration, 282(3-5), 701-712, (2005).

[23] M. Tucsnak, Regularity and exact controllability for a beam with piezoelectric actuator, SIAM J. Control Optim., 34, 922-930 (1996). 